\title{
Mixed models for optimal saving with labor income risk and interest-rate risk
}

\author{
Irina Georgescu ${ }^{1}$ (1)
}

Accepted: 12 January 2022 / Published online: 31 January 2022

(c) The Author(s), under exclusive licence to Springer-Verlag GmbH Germany, part of Springer Nature 2022

\begin{abstract}
In this paper, optimal saving models with two risk components are studied: the labor income risk and the interest rate risk. These risks can be modeled probabilistically by random variables or possibilistically by fuzzy numbers. In mixed models, one of the components is probabilistic and the other one is possibilistic. After the construction of two mixed models of optimal saving, several notions of precautionary saving are defined. These measure the variation in optimal saving level when moving from one model to another (usually by adding a risk component). The main results of the paper establish necessary and sufficient conditions on the positivity of different precautionary savings. (This means that the presence of new risks generates extra-saving.)
\end{abstract}

Keywords Mixed expected utility $\cdot$ Optimal saving $\cdot$ Labor income risk $\cdot$ Interest rate risk $\cdot$ Precautionary saving

\section{Introduction}

The saving is an important component of a consumer activity. An agent (consumer) allocates a part of his wealth to cope in future with risk losses. The saving activity is analyzed usually by two-period models, denoted by 0 and 1 , in which risk appears in period 1 . In period 0 , the agent's wealth $y_{0}$ is divided between consumption and saving: a part $s$ of $y_{0}$ is invested in the financial market. The investment $s$ will generate in period 1 a return $s(1+r)$ (where $r$ is the interest rate for saving), which will mitigate the loss of risk. The purpose of the agent is to find that level of $s$ maximizing the total utility of the model.

The first papers that investigated the effects of risk on the optimal saving have stopped on two types of risk: the labor income risk (Leland 1968; Sandmo 1970; Drèze and Modigliani 1972) and the interest-rate risk (Sandmo 1970; Rothschild and Stiglitz 1971). The notion of precautionary saving has been introduced as a measure of the variation in optimal saving when moving from a certain model to a risk model. The positivity of precautionary saving signifies the

Irina Georgescu

irina.georgescu@csie.ase.ro

1 Department of Informatics and Economic Cybernetics Bucharest, University of Economics, Piata Romana No 6 R

70167, Oficiul Postal 22, Bucharest, Romania increase in optimal saving level when a risk appears. The mentioned authors have focused their research on finding some necessary and sufficient conditions on the positivity of optimal saving. In case of labor income risk, such a necessary and sufficient condition is that consumer's prudence (according to Kimball 1990, 1992) is equivalent to the positivity of the third derivative of the consumer's utility function). For models with interest-rate risk, the precautionary saving is positive if and only if the relative prudence index (Kimball 1992) is higher than 2 (by a Rothschild and Stiglitz theorem from Rothschild and Stiglitz 1971).

The labor income risk and the interest-rate risk appear as separate risks also in Eeckhoudt and Schlesinger (2008) where there are analyzed the effects of the $n$-th-order changes on the optimal saving. The paper Magnani (2017) contains a new proof of the Rothschild and Stiglitz theorem from Rothschild and Stiglitz (1971), as well as its new interpretation.

We will note that there are other economic themes that are studied using saving models: among them are bequest motives (see Jappelli and Pistaferri 2017, Section 12) and smoothing consumption (Kimball and Weil 2009). The paper Vergara and Bonilla (2021) investigates the precautionary saving in a mean-variance model (where the variance is taken as the measure of uncertainty); then, the results are applied to some decision problems under risk (including the economic effects given by COVID-19 pandemic). 
Papers Baiardi et al. (2014), Li (2012), Gunning (2010) and Vergara (2007) deal with saving models in which labor income risk and interest rate risk appear together, setting necessary and sufficient conditions for the presence of these risks to generate extra-saving. The first saving model with background risk has been studied by Eeckhoudt et al. (2007). Among the papers treating saving models with more parameters, we remember the works Courbage and Rey (2007), Magnani and Menegatti (2015) and Nocetti (2016). A state of the art dedicated to precautionary saving can be found in the recent paper by Baiardi et al. (2019).

In all these papers, risk is modelled by random variables, and the saving model construction is done in the framework of Von Neumann-Morgenstern EU theory. Probabilistic uncertainty modeling does not cover all the phenomena in which uncertainty occurs (a typical situation is when information is extracted from a set of insufficiently broad data). Several papers from the last two decades treat the uncertainty phenomenon by Zadeh's possibility theory (Zadeh 1978). According to Dubois et al. (2004), p. 277, "while probability theory offers a quantitative model for randomness and indecisiveness, possibility theory offers a qualitative model of incomplete knowledge". In probabilistic modeling, the risk is represented by a possibility distribution (in particular, by a fuzzy number), and the models are built in a possibilistic EU theory (by Carlsson and Fullér 2011; Dubois and Prade 1988; Georgescu 2012, 2009, 2011, etc.). In the study by possibilistic methods of the different themes of the risk theory are used possible indicators attached to the fuzzy numbers: possibilistic expected values, various notions of possibilistic variance, possibilistic moments, etc. (Carlsson and Fullér 2011; Georgescu 2012).

Some possibilistic models for optimal saving have been defined in Lucia-Casademunt and Georgescu (2013), Georgescu (2014), Georgescu and Kinnunen (2016) and Georgescu and Kinnunen (2020). In Lucia-Casademunt and Georgescu (2013) we studied a model in which the labor income risk is a fuzzy number, and in Georgescu and Kinnunen (2020) a model in which the interest-rate risk is a fuzzy number. A treatment of the case in which risk appears as a bidimensional vector, in which both labor income risk and interest-rate risk are fuzzy numbers can be found in Georgescu and Kinnunen (2016).

This paper is concerned with two models of saving in which risk is a mixed bidimensional vector: one component is a random variable, the other is a fuzzy number. The model construction is realized in a "mixed EU-theory", in which the main concept is the mixed expected utility introduced in Georgescu (2011). The combined effect of mixed risk leads to new notions of optimal saving, measuring the optimal saving variation from the certain model to each of the two mixed models.

We will expose shortly the content of the paper.
Section 2 presents the possibilistic expected utility associated with a weighting function, a fuzzy number and a unidimensional utility function (by Georgescu 2012, 2011). The two main possibilistic indicators associated with a fuzzy number (the expected value and the variance) are recalled. Section 3 contains the definition of mixed expected utility (associated with a weighting function, a mixed vector and a bidimensional utility function) and some of its properties (by Georgescu 2012, Chapter 7 and Georgescu 2014).

In Sect. 4 there are recalled from Eeckhoudt et al. (2005), Eeckhoudt and Schlesinger (2008), Lucia-Casademunt and Georgescu (2013) and Georgescu and Kinnunen (2020) and five optimal saving models (the certain model, two probabilistic models and two more possibilistic models) together with the first-order conditions.

Section 5 deals with the construction of the two mixed optimal saving models. In the first model, the labor income risk is a fuzzy number; in the second model the labor income risk is a random variable and the interest-rate risk is a fuzzy number. Their total utility functions are defined, their concavity is proved and the first-order conditions are written.

In Sect. 6, the precautionary saving notions of the two mixed models are defined and necessary and sufficient conditions for the mixed risk to generate extra-saving are established. These extra-saving conditions are expressed in terms of the partial relative prudence index from Baiardi et al. (2014) and two new indicators $K_{1}, K_{2}$. We prove some approximation formulas for the computation of the indicators $K_{1}, K_{2}$.

These four notions of precautionary saving are introduced in Sect. 7. They measure the variation in optimal saving when moving from the single risk models from Sect. 4 to the two mixed models from Sect. 5. The main results of the section contain necessary and sufficient conditions for the positivity of the new precautionary savings.

In Sect. 8, we prove that the optimal savings corresponding to the two mixed models studied in this paper can be calculated as the real solutions of some third-degree equations.

The sample percentile method of Vercher et al. (2007) is used in Sect. 9 for obtaining the values of indicators $K_{1}$ and $K_{2}$ starting from a dataset.

\section{Possibilistic expected utility}

If $(\Omega, \mathcal{K}, P)$ is a probability space and $X: \Omega \rightarrow \mathbf{R}$ is a random variable, then we will denote by $M(X)$ the mean value of $X$ and by $\operatorname{Var}(X)$ its variance. Let $u: \mathbf{R} \rightarrow \mathbf{R}$ a continuous utility function, representing an agent (consumer). Then, $u(X)=u \circ X$ is a random variable and its mean value $M(u(X))$ is called the expected utility associated with $X$ and $u$. In the probabilistic risk modeling (Eeckhoudt et al. 
2005; Gollier 2004), two basic concepts exist: a utility function $u$ (of an agent) and a random variable $X$ representing the risk. The treatment of different risk topics takes place in the framework of Von Neumann-Morgenstern EU-theory, whose fundamental notion is the (probabilistic) expected utility $M(u(X))$.

The possibility risk theory (Georgescu 2012, 2011; Georgescu and Kinnunen 2016) is developed in a framework based on the following three entities:

- a weighting function $f:[0,1] \rightarrow \mathbf{R}(f$ is a nonnegative and increasing function that satisfies $\left.\int_{0}^{1} f(\gamma) d \gamma=1\right)$.

- a fuzzy number $A$ whose level sets are $[A]^{\gamma}=$ $\left[a_{1}(\gamma), a_{2}(\gamma)\right], \gamma \in[0,1]$

- a utility function of class $\mathcal{C}^{2}$.

Risk is modeled by a fuzzy number $A$ and $u$ represents the agent (consumer). In general, the possibilistic risk can be modeled by a possibility distribution. The choice of fuzzy numbers to represent the possibilistic risk is due to the fact that they can satisfactorily model many situations of uncertainty (see Dubois and Prade 1988; Carlsson and Fullér 2011). We will also notice that the set of fuzzy numbers has a rich algebraic structure (Dubois and Prade 1988), which allows the development of a mathematical apparatus capable of modeling the themes of risk theory.

In order to be able to develop a EU-theory in the possibilistic context fixed by the three entities, we need a concept of possibilistic expected utility.

Definition 2.1 (Georgescu 2012, 2011) The possibilistic expected utility $E_{f}(u(A))$ associated with the triple $(f, A, u)$ is defined by:

$E_{f}(u(A))=\frac{1}{2} \int_{0}^{1}\left[u\left(a_{1}(\gamma)\right)+u\left(a_{2}(\gamma)\right)\right] f(\gamma) d \gamma$

For some particular forms of the utility function $u$ from (2.1), we could reach the main indicators associated with the fuzzy number $A$.

When in (2.1) we take $u=1_{\mathbf{R}}$ (the identity of $\mathbf{R}$ ), we obtain the possibilistic expected value:

$E_{f}(A)=\frac{1}{2} \int_{0}^{1}\left[a_{1}(\gamma)+a_{2}(\gamma)\right] f(\gamma) d \gamma$

For $u(x)=\left(x-E_{f}(A)\right)^{2}$, one obtains the possibilistic variance

$\operatorname{Var}_{f}(A)=\frac{1}{2} \int_{0}^{1}\left[\left(a_{1}(\gamma)-E_{f}(A)\right)^{2}+\left(a_{2}(\gamma)-E_{f}(A)\right)^{2}\right]$
$f(\gamma) d \gamma$
In the particular case of the weighting function $f(\gamma)=$ $2 \gamma, \gamma \in[0,1]$, the notions of possibilistic expected utility and possibilistic variance have been introduced by Carlsson and Fullér in paper Carlsson and Fullér (2001).

We will notice that in Georgescu (2009) was defined another notion of possibilistic expected utility: it corresponds to the same possibilistic expected value, but to a different possibilistic variance.

Remark 2.2 The support of the fuzzy number $A$ is $\operatorname{supp}(A)=$ $\{x \in \mathbf{R} \mid A(x)>0\}$. If $\operatorname{supp}(A) \subseteq(0, \infty)$, then $E_{f}(A)>0$. For any fuzzy number $A$ whose support is not a point set, we have $\operatorname{Var}_{f}(A)>0$.

Proposition 2.3 (Georgescu 2012, 2011) For $a, b \in \mathbf{R}, g$ : $\mathbf{R} \rightarrow \mathbf{R}, h: \mathbf{R} \rightarrow \mathbf{R}$ two utility functions and $u=a g+b h$. Then $E_{f}(u(A))=a E_{f}(g(A))+b E_{f}(h(A))$.

The linearity property of Proposition 2.3 allows the proof of an approximation formula for $E_{f}(u(A))$, analogous to the Arrow-Pratt approximation formula from the possibility risk theory (Eeckhoudt et al. 2005, p. 11).

Proposition 2.4 (Georgescu 2012, 2011) For any fuzzy number $A$ and for any utility function $u$ (of class $\mathcal{C}^{2}$ ), the following approximation formula holds:

$E_{f}(u(A)) \approx u\left(E_{f}(A)\right)+\frac{1}{2} u^{\prime \prime}\left(E_{f}(A)\right) \operatorname{Var}_{f}(A)$

\section{Mixed expected utility}

Mixed vectors describe situations with several risk parameters: some of them are fuzzy numbers, others are random variables. In particular, a mixed bidimensional vector has the form $(A, X)$ or $(X, A)$, where $A$ is a fuzzy number and $X$ a random variable. Such mixed bidimensional vectors will appear in the saving models from Sects. 5-7: a component of the mixed vector will be the labor income risk and the other component will be the interest rate risk. We fix a mixed vector $(A, X)$. Assume that the level sets of the fuzzy number $A$ are $[A]^{\gamma}=\left[a_{1}(\gamma), a_{2}(\gamma)\right], \gamma \in[0,1]$. Let $f:[0,1] \rightarrow \mathbf{R}$ be a weighting function and $u: \mathbf{R}^{2} \rightarrow \mathbf{R}$ a bidimensional utility function of class $\mathcal{C}^{2}$.

Definition 3.1 (Georgescu 2012, 2011) The mixed expected utility $E_{f}(u(A, X))$ associated with the triple $(f,(A, X), u)$ is defined by

$$
\begin{aligned}
E_{f}(u(A, X))= & \frac{1}{2} \int_{0}^{1}\left[M\left(u\left(a_{1}(\gamma), X\right)\right)\right. \\
& \left.+M\left(u\left(a_{2}(\gamma), X\right)\right)\right] f(\gamma) d \gamma
\end{aligned}
$$


in which for any $i=1,2$ and $\gamma \in[0,1], M\left(u\left(a_{i}(\gamma), X\right)\right)$ is the mean value associated with the random variable $u\left(a_{i}(\gamma), X\right)$.

Analogously, one defines the mixed expected utility $E_{f}(u(X, A))$ associated with $f$, the mixed vector $(X, A)$ and the utility function $u$.

Proposition 3.2 (Georgescu 2012, 2011) Let $g, h$ be two bidimensional utility functions, $a, b \in \mathbf{R}$ and $u=a g+b h$. Then, $E_{f}(u(A, X))=a E_{f}(g(A, X))+b E_{f}(h(A, X))$.

If $u$ is a bidimensional utility function of class $\mathcal{C}^{2}$, then we denote as usual by $u_{i}, u_{i j}$ the first and the second partial derivatives of $u$.

The linearity property from Proposition 3.2 and the second-order Taylor approximation applied to the utility function $u$ are used in proving the approximation formula of the mixed expected utility $E_{f}(u(X, A))$ contained in the following result:

Proposition 3.3 (Georgescu 2012, 2011) Let $(A, X)$ be a mixed vector and $u: \mathbf{R}^{2} \rightarrow \mathbf{R}$ a bidimensional utility function of class $\mathcal{C}^{2}$. Then, the following approximation formula holds:

$$
\begin{gathered}
E_{f}(u(A, X)) \approx u\left(E_{f}(A), M(X)\right) \\
+\frac{1}{2} u_{11}\left(E_{f}(A), M(X)\right) \operatorname{Var}_{f}(A) \\
+\frac{1}{2} u_{22}\left(E_{f}(A), M(X)\right) \operatorname{Var}(X)
\end{gathered}
$$

The formula from the previous proposition approximates $E_{f}(u(A, X))$ according to the probabilistic indicators $M(X)$, $\operatorname{Var}(X)$ and the possibilistic indicators $E_{f}(A), \operatorname{Var}_{f}(A)$. It will be used in the following sections to find some approximation formulas of the total utility functions corresponding to the mixed saving models.

\section{Probabilistic and possibilistic models of optimal saving}

In this section, there are presented five optimal saving models. To each model, one associates an optimization problem whose solution represents the saving level for which the total utility of the model is maximum. We will start by defining the certain model (without risk), which will be then transformed into models with one of the two types or risk: labor income risk and interest-rate risk. These will be mathematically described by random variables or fuzzy numbers.

Certain model (Eeckhoudt et al. 2005, p. 96 or Georgescu 2009) We consider a two-period consumption model: the two periods will be denoted by 0 and 1 . The model is defined by the following entities:
- the consumer has the same utility function $u(x)$ for both periods ${ }^{1}$;

- in both periods 0 and 1 there are the sure incomes $y_{0}$ and $y_{1}$, respectively;

- $r$ is the interest rate for saving;

- $R=1+r>0$ is the return of saving (the gross rate of interest, in the terminology of Eeckhoudt and Schlesinger 2008);

- $s>0$ is the level of saving.

As usual, we will assume that the utility function is of class $\mathcal{C}^{2}$ and $u^{\prime}>0, u^{\prime \prime}<0$. The total utility function of consumption will be:

$U(s)=u\left(y_{0}-s\right)+u\left(y_{1}+s R\right)$

The function $U$ is strictly concave. The consumer wishes to determine that value $s^{*}$ of $s$ maximizing the total utility $U(s)$ :

$\max _{s} U(s)=U\left(s^{*}\right)$

The first-order condition associated with (4.2) will be:

$-u^{\prime}\left(y_{0}-s\right)+R u^{\prime}\left(y_{1}+R s\right)=0$

To obtain saving models with risk, instead of parameters $y_{1}$ and $R$ we will consider random variables or fuzzy numbers. We fix a weighting function $f:[0,1] \rightarrow \mathbf{R}$.

Probabilistic model with labor income risk (Eeckhoudt et al. 2005, pp. 95-98 or Eeckhoudt and Schlesinger 2008)

In period 1 , instead of $y_{1}$ we will have a labor income risk represented by a random variable $\tilde{y}$ with $M(\tilde{y})=y_{1}$. The total utility function will be:

$V_{1}(s)=u\left(y_{0}-s\right)+M[u(\tilde{y}+R s)]$

The solution of the optimal problem $\max _{s} V_{1}(s)$ will be denoted $s_{1}^{*}$, and the first-order condition is written

$-u^{\prime}\left(y_{0}-s\right)+R M\left[u^{\prime}(\tilde{y}+R s)\right]=0$

Probabilistic model with interest-rate risk (Eeckhoudt et al. 2005, p. 98-99 or Eeckhoudt and Schlesinger 2008)

In period 1, instead of $r$ we have an interest-rate risk represented by a random variable $\tilde{r}$ with the property $M(\tilde{r})=r$. Thus, the gross rate of interest will be the random variable

\footnotetext{
1 The treatment of the case when the utility functions are distinct is done similarly.
} 
Table 1 Four models with risk

\begin{tabular}{lllll}
\hline No. & Labor income & Interest rate & Total utility function & Optimal saving \\
\hline 1 & $\tilde{y}$ & $R$ & $V_{1}(s)$ & $s_{1}^{*}$ \\
2 & $y_{1}$ & $\tilde{R}$ & $V_{2}(s)$ & $s_{2}^{*}$ \\
3 & $A$ & $R$ & $V_{3}(s)$ & $s_{3}^{*}$ \\
4 & $y_{1}$ & $B$ & $V_{4}(s)$ & $s_{4}^{*}$ \\
\hline
\end{tabular}

$\tilde{R}=1+\tilde{r}$; therefore, $M(\tilde{R})=1+M(\tilde{r})=1+r=R$. The total utility function will be:

$V_{2}(s)=u\left(y_{0}-s\right)+M\left[u\left(y_{1}+s \tilde{R}\right)\right]$

The solution of the optimization problem $\max _{s} V_{2}(s)$ will be denoted $s_{2}^{*}$, and the first-order condition is written:

$-u^{\prime}\left(y_{0}-s\right)+M\left[\tilde{R} u^{\prime}\left(y_{1}+s \tilde{R}\right)\right]=0$

Possibilistic model with labor income risk (Lucia-Casademun and Georgescu 2013)

In period 1 , instead of $y_{1}$ we will have a labor income risk represented by a fuzzy number $A$ with $E_{f}(A)=y_{1}$. The total utility function will be:

$V_{3}(s)=u\left(y_{0}-s\right)+E_{f}[u(A+R s)]$

$V_{3}$ is a strictly concave function (Lucia-Casademunt and Georgescu 2013). We denote by $s_{3}^{*}$ the solution of the problem $\max _{s} V_{3}(s)$. The first-order condition has the following form:

$-u^{\prime}\left(y_{0}-s\right)+R E_{f}\left[u^{\prime}(A+R s)\right]=0$

Possibilistic model with interest-rate risk (Georgescu and Kinnunen 2020)

In period 1 , instead of $y_{1}$ we will have a return of saving represented by a fuzzy number $B$ with $E_{f}(B)=R$. The total utility function will be:

$V_{4}(s)=u\left(y_{0}-s\right)+E_{f}\left[u\left(y_{1}+s B\right)\right]$

$V_{4}$ is a strictly concave function (Georgescu and Kinnunen 2020). We denote by $s_{4}^{*}$ the solution of the optimization problem $\max _{s} V_{4}(s)$. We consider the function

$m(s, y, x)=u(y+s x)$

By Georgescu and Kinnunen (2020), the first-order condition associated with the problem $\max _{s} V_{4}(s)$ has the form:

$-u^{\prime}\left(y_{0}-s\right)+E_{f}\left[\frac{\partial m(s, y, B)}{\partial s}\right]=0$
Table 2 Models with two types of risk

\begin{tabular}{lll}
\hline No. & Labor income risk & Interest rate risk \\
\hline 1 & Probabilistic & Probabilistic \\
2 & Possibilistic & Possibilistic \\
3 & Possibilistic & Probabilistic \\
4 & Probabilistic & Possibilistic \\
\hline
\end{tabular}

where $E_{f}\left[\frac{\partial m(s, y, B)}{\partial s}\right]$ is the possibilistic expected utility associated with $f$, the fuzzy number $B$ and a utility function $\frac{\partial m(s, y, B)}{\partial s}$.

We summarize the above information on the four models with risk in Table 1.

In defining the total utility functions of the possibilistic saving models and the first-order conditions associated with them the possibilistic expected utilities have been used in the sense of Definition 2.1.

The four models from Table 1 will be denoted $(\tilde{y}, R)$, $\left(y_{1}, \tilde{R}\right),(A, R)$ and $\left(y_{1}, B\right)$.

Remark 4.1 Throughout this paper, we will make the following assumptions on the fuzzy numbers $A, B$ and on the random variables $\tilde{y}, \tilde{R}$ :

$(H) \operatorname{supp}(A), \operatorname{supp}(B), \operatorname{supp}(\tilde{y})=\{\omega \in \Omega \mid \tilde{y}(\omega)>0\}$ and $\operatorname{supp}(\tilde{R})=\{\omega \in \Omega \mid \tilde{R}(\omega)>0\}$ are subsets of $(0, \infty)$.

By $(H)$, it follows that $E_{f}(A)>0, E_{f}(B)>0, M(\tilde{y})>$ $0, M(\tilde{R})>0$, which agrees with $y_{1}>0, R>0, E_{f}(A)=$ $M(\tilde{y})=y_{1}$ and $E_{f}(B)=M(\tilde{R})=R$.

\section{Mixed models of optimal saving}

In this section, we will consider optimal saving models in which labor income risk and interest rate risk are jointly present. According to the type of modeling of these (probabilistic or possibilistic) risks, four situations are distinguished as follows:

The models of type 1 have been studied in Baiardi et al. (2014) and models of type 2 in Georgescu and Kinnunen (2016). We will expose next the construction of mixed models of types 3 and 4 . The total utility functions of the two mixed saving models will be defined using the mixed expected utilities in the sense of Definition 3.1. 
Models of type 3 These optimal saving models are characterized by the fact that the labor income risk is a fuzzy number $A$ with $E_{f}(A)=y_{1}$ and the return of saving is a random variable $\tilde{R}$ with $M(\tilde{R})=R$.

The total utility function of the model will be:

$V_{5}(s)=u\left(y_{0}-s\right)+E_{f}[u(A+s \tilde{R})]$

in which $E_{f}[u(A+s \tilde{R})]$ is the mixed expected utility associated with $f$, the mixed vector $(A, \tilde{R})$ and the bidimensional utility function $g(y, x)=u(y+s x)$, in which $s$ is a parameter. We denote by $[A]^{\gamma}=\left[a_{1}(\gamma), a_{2}(\gamma)\right]$ the level sets of the fuzzy number $A$. By (5.1), the total utility $V_{5}(s)$ will be written:

$$
\begin{aligned}
V_{5}(s)= & u\left(y_{0}-s\right)+\frac{1}{2} \int_{0}^{1}\left[M\left(u\left(a_{1}(\gamma)+s \tilde{R}\right)\right)\right. \\
& \left.+M\left(u\left(a_{2}(\gamma)+s \tilde{R}\right)\right)\right] f(\gamma) d \gamma
\end{aligned}
$$

By derivation, one obtains

$$
\begin{aligned}
V_{5}^{\prime}(s)= & -u^{\prime}\left(y_{0}-s\right)+\frac{1}{2} \int_{0}^{1}\left[M\left(\tilde{R} u^{\prime}\left(a_{1}(\gamma)+s \tilde{R}\right)\right)\right. \\
& \left.+M\left(\tilde{R} u^{\prime}\left(a_{2}(\gamma)+s \tilde{R}\right)\right)\right] f(\gamma) d \gamma
\end{aligned}
$$

We consider the function

$w(y, x, s)=x u^{\prime}(y+s x)$

Then, by Definition 3.1 the above expression of $V_{5}^{\prime}(s)$ will be written concisely:

$V_{5}^{\prime}(s)=-u^{\prime}\left(y_{0}-s\right)+E_{f}[w(A, \tilde{R}, s)]$

Proposition $5.1 \quad V_{5}$ is a strictly concave function.

Proof We derive $V_{5}^{\prime}(s)$ :

$$
\begin{aligned}
V_{5}^{\prime \prime}(s)= & u^{\prime \prime}\left(y_{0}-s\right)+\frac{1}{2} \int_{0}^{1}\left[M\left((\tilde{R})^{2} u^{\prime \prime}\left(a_{1}(\gamma)+s \tilde{R}\right)\right)\right. \\
& \left.+M\left((\tilde{R})^{2} u^{\prime \prime}\left(a_{2}(\gamma)+s \tilde{R}\right)\right)\right] f(\gamma) d \gamma
\end{aligned}
$$

Since $u^{\prime \prime}<0$ by hypothesis, it follows $V_{5}^{\prime \prime}(s)<0$ for any $s$.

We denote by $s_{5}^{*}$ the solution of the optimization problem:

$$
\max _{s} V_{5}(s)
$$

The value $s_{5}^{*}$ is the optimal saving level in the presence of the mixed risk $(A, \tilde{R})$. By (5.3), the first-order condition $s_{5}^{*}=0$ is written:

$$
-u^{\prime}\left(y_{0}-s\right)+E_{f}[w(A, \tilde{R}, s)]=0
$$

Models of type 4 . In these optimal saving models, the labor income risk is a random variable $\tilde{y}$ with $M(\tilde{y})=y_{1}$ and the return of saving is a fuzzy number $B$ with $E_{f}(B)=R$.

The total utility function $V_{6}(s)$ of this model is:

$V_{6}(s)=u\left(y_{0}-s\right)+E_{f}[u(\tilde{y}+s B)]$

in which $E_{f}[u(\tilde{y}+s B)]$ is the mixed expected utility associated with the weighting function $f$, the mixed vector $(\tilde{y}, B)$ and the utility function $g(y, x)=u(y+s x)$.

Assume that the level sets of the fuzzy number $B$ are $[B]^{\gamma}=\left[b_{1}(\gamma), b_{2}(\gamma)\right], \gamma \in[0,1]$. By (3.1), the definition (5.6) of $V_{6}(s)$ can be written:

$$
\begin{aligned}
V_{6}(s)= & u\left(y_{0}-s\right)+\frac{1}{2} \int_{0}^{1}\left[M\left(u\left(\tilde{y}+s b_{1}(\gamma)\right)\right)\right. \\
& \left.+M\left(u\left(\tilde{y}+s b_{2}(\gamma)\right)\right)\right] f(\gamma) d \gamma
\end{aligned}
$$

from where, by derivation, one obtains:

$$
\begin{aligned}
V_{6}^{\prime}(s)= & -u^{\prime}\left(y_{0}-s\right)+\frac{1}{2} \int_{0}^{1}\left[b_{1}(\gamma) M\left(u^{\prime}\left(\tilde{y}+s b_{1}(\gamma)\right)\right)\right. \\
& \left.+b_{2}(\gamma) M\left(u^{\prime}\left(\tilde{y}+s b_{2}(\gamma)\right)\right)\right] f(\gamma) d \gamma
\end{aligned}
$$

Then, by (3.1), one obtains:

$$
V_{6}^{\prime}(s)=-u^{\prime}\left(y_{0}-s\right)+E_{f}[w(\tilde{y}, B, s)]
$$

where $w$ is the function defined in (5.2).

Proposition 5.2 $V_{6}$ is a strictly concave function.

Proof Analogously to the proof of Proposition 5.1, it is proved that $V^{\prime \prime}(s)<0$ for any $s$.

We denote by $s_{6}^{*}$ the solution of the following optimization problem:

$\max _{s} V_{6}(s)$

By (5.7), the first-order condition associated with (5.8) is written

$$
-u^{\prime}\left(y_{0}-s\right)+E_{f}[w(\tilde{y}, B, s)]=0
$$

Remark 5.3 By Propositions 5.1 and 5.2, the total utility functions $V_{5}$ and $V_{6}$ are strictly concave; thus, the derivatives $V_{5}^{\prime}$ and $V_{6}^{\prime}$ are strictly decreasing. The optimal savings $s_{5}^{*}$ and $s_{6}^{*}$ are solutions of the first-order conditions $V_{5}^{\prime}(s)=0$ and $V_{6}^{\prime}(s)=0$. Accordingly, when these solutions exist, they are unique.

For the convenience of writing, the model of type 3 will be symbolized by the mixed vector $(A, \tilde{R})$, and the model of type 4 by $(\tilde{y}, B)$. 


\section{From certain model to mixed models}

Traditionally, the precautionary saving measures the variation in optimal saving when the certain model is transformed into an optimal saving model in which a certain type of risk is present. By Baiardi et al. (2019), an important part of the results from the literature dedicated to the saving models contains necessary and sufficient conditions (either only necessary or only sufficient) for the presence of a certain type of risk to generate extra-saving. The main results of the section belong to this research stream, necessary and sufficient conditions being established for the extra-saving in case of the two mixed models defined in Sect. 5.

In this section, we will define two new notions of precautionary saving. They indicate how the optimal saving level increases or decreases when moving from the certain model (4.2) to each of the two mixed models $(A, \tilde{R})$ and $(\tilde{y}, B)$ defined in the previous section.

We will keep all the notations from Sects. 4 and 5. We fix a weighting function $f:[0,1] \rightarrow \mathbf{R}$.

We define now the two notions of precautionary saving:

- $s_{5}^{*}-s^{*}$ : the variation in optimal saving when moving from the certain model to the mixed model $(A, \tilde{R})$;

- $s_{6}^{*}-s^{*}$ : the variation in optimal saving when moving from the certain model to the mixed model $(\tilde{y}, B)$.

We intend in the following to find necessary and sufficient conditions for the positivity of those two precautionary savings. We recall from Baiardi et al. (2014) the partial relative prudence index of the utility function $u$ :

$P R P(y, x)=-x \frac{u^{\prime \prime \prime}(y+x)}{u^{\prime \prime}(y+x)}, x>0$

Also, similar to Baiardi et al. (2014) (18) we introduce the following new indicators:

$K_{1}=2 \frac{\left(s^{*}\right)^{2} \operatorname{Var}(\tilde{R})}{\operatorname{Var}_{f}(A)+\left(s^{*}\right)^{2} \operatorname{Var}(\tilde{R})}$ for the $\operatorname{model}(A, \tilde{R})$

$K_{2}=2 \frac{\left(s^{*}\right)^{2} \operatorname{Var}_{f}(B)}{\operatorname{Var}(\tilde{y})+\left(s^{*}\right)^{2} \operatorname{Var}_{f}(B)}$ for the $\operatorname{model}(\tilde{y}, B)$

The indicator $K_{1}$ depends on the possibilistic variance $\operatorname{Var}_{f}(A)$, the probabilistic variance $\operatorname{Var}(\tilde{R})$ and the level of optimal saving $s^{*}$ for the certain model; similarly, $K_{2}$ depends on $\operatorname{Var}_{f}(B), \operatorname{Var}(\tilde{y})$ and $s^{*}$.
The indicators $K_{1}$ and $K_{2}$ can be written as:

$$
K_{1}=\frac{2}{1+\frac{\operatorname{Var}_{f}(A)}{\left(s^{*}\right)^{2} \operatorname{Var}(\tilde{R})}} ; K_{2}=\frac{2}{1+\frac{\left.\operatorname{Var}_{(\tilde{y}}\right)}{\left(s^{2}\right)^{2} \operatorname{Var}_{f}(B)}}
$$

Then, the following equivalences follow:

- $K_{1} \leq 2$ iff $s^{*} \leq \frac{\operatorname{Var}_{f}(A)}{\operatorname{Var}(\tilde{R})}$ and $K_{2} \leq 2$ iff $s^{*} \leq \frac{\operatorname{Var}(\tilde{y})}{\operatorname{Var}(B)}$;

- $K_{1}=2$ iff $s^{*}=\frac{\operatorname{Var}_{f}(A)}{\operatorname{Var}(\tilde{R})}$ and $K_{2}=2$ iff $s^{*}=\frac{\operatorname{Var}(\tilde{y})}{\operatorname{Var}_{f}(B)}$.

- For the rest of the section, we will assume that $\operatorname{supp}(A)$ and $\operatorname{supp}(B)$ are not point sets; thus, by Remark 2.2, we will have $\operatorname{Var}_{f}(A)>0$ and $\operatorname{Var}_{f}(B)>0$. Then from (6.2) and (6.3), it follows $K_{1}>0$ and $K_{2}>0$.

Theorem $6.1 s_{5}^{*}-s^{*}>0$ ifand only if $K_{1}<P R P\left(y_{1}, s^{*} R\right)$.

Proof We consider the following bidimensional function:

$v(y, x)=w\left(y, x, s^{*}\right)=x u^{\prime}\left(y+s^{*} x\right)$

By (5.3), we will have

$$
\begin{aligned}
V_{5}^{\prime}\left(s^{*}\right) & =-u^{\prime}\left(y_{0}-s^{*}\right)+E_{f}\left[w\left(A, \tilde{R}, s^{*}\right)\right] \\
& =-u^{\prime}\left(y_{0}-s^{*}\right)+E_{f}[v(A, \tilde{R})]
\end{aligned}
$$

But, according to the first-order condition (4.3) we have $u^{\prime}\left(y_{0}-s^{*}\right)=R u^{\prime}\left(y_{1}+s^{*} R\right)$; thus, $V_{5}^{\prime}\left(s^{*}\right)$ can be written

$V_{5}^{\prime}\left(s^{*}\right)=-R u^{\prime}\left(y_{1}+s^{*} R\right)+E_{f}[v(A, \tilde{R})]$

By applying the approximation formula from Proposition 3.3, it follows

$$
\begin{gathered}
E_{f}[v(A, \tilde{R})] \approx v\left(E_{f}(A), M(\tilde{R})\right) \\
+\frac{1}{2} v_{11}\left(E_{f}(A), M(\tilde{R})\right) \operatorname{Var}_{f}(A) \\
+\frac{1}{2} v_{22}\left(E_{f}(A), M(\tilde{R})\right) \operatorname{Var}(\tilde{R})
\end{gathered}
$$

Since $E_{f}(A)=y_{1}$ and $M(\tilde{R})=R$, the previous formula is written:

$$
\begin{aligned}
& E_{f}[v(A, \tilde{R})] \approx v\left(y_{1}, R\right)+\frac{1}{2} v_{11}\left(y_{1}, R\right) \operatorname{Var}_{f}(A) \\
& \quad+\frac{1}{2} v_{22}\left(y_{1}, R\right) \operatorname{Var}(\tilde{R})
\end{aligned}
$$


We notice that

$$
\begin{aligned}
& v_{1}(y, x)=x u^{\prime \prime}\left(y+s^{*} x\right) ; v_{11}(y, x)=x u^{\prime \prime \prime}\left(y+s^{*} x\right) \\
& \begin{aligned}
v_{2}(y, x) & =\frac{\partial}{\partial x}\left[x u^{\prime}\left(y+s^{*} x\right)\right] \\
= & u^{\prime}\left(y+s^{*} x\right)+s^{*} x u^{\prime \prime}\left(y+s^{*} x\right)
\end{aligned} \\
& v_{22}(y, x)=\frac{\partial}{\partial x}\left[u^{\prime}\left(y+s^{*} x\right)+s^{*} x u^{\prime \prime}\left(y+s^{*} x\right)\right] \\
& =s^{*} u^{\prime \prime}\left(y+s^{*} x\right)+s^{*}\left[u^{\prime \prime}\left(y+s^{*} x\right)+x u^{\prime \prime \prime}\left(y+s^{*} x\right)\right] \\
& =2 s^{*} u^{\prime \prime}\left(y+s^{*} x\right)+\left(s^{*}\right)^{2} x u^{\prime \prime \prime}\left(y+s^{*} x\right)
\end{aligned}
$$

Replacing $v_{11}\left(y_{1}, R\right)$ and $v_{22}\left(y_{1}, R\right)$ in (6.6) with the values given by the above computations, one obtains:

$$
\begin{gathered}
E_{f}[v(A, \tilde{R})] \approx v\left(y_{1}, R\right)+\frac{\operatorname{Var}_{f}(A)}{2} R u^{\prime \prime \prime}\left(y_{1}+s^{*} R\right) \\
+\frac{\operatorname{Var}(\tilde{R})}{2} s^{*}\left[2 u^{\prime \prime}\left(y_{1}+s^{*} R\right)+s^{*} R u^{\prime \prime \prime}\left(y_{1}+s^{*} R\right)\right]
\end{gathered}
$$

We notice that by (6.4) we get $v\left(y_{1}, R\right)=R u^{\prime}\left(y_{1}+\right.$ $\left.s^{*} R\right)$. Then, taking into account the above approximation of $E_{f}[v(A, \tilde{R})]$ one obtains

$$
\begin{aligned}
V_{5}^{\prime}\left(s^{*}\right)= & -u^{\prime}\left(y_{0}-s^{*}\right)+E_{f}[v(A, R)] \approx \\
\approx & -R u^{\prime}\left(y_{1}+s^{*} R\right)+R u^{\prime}\left(y_{1}+s^{*} R\right) \\
& +\frac{\operatorname{Var}_{f}(A)}{2} R u^{\prime \prime \prime}\left(y_{1}+s^{*} R\right) \\
& +\frac{\operatorname{Var}(\tilde{R})}{2} s^{*}\left[2 u^{\prime \prime}\left(y_{1}+s^{*} R\right)+s^{*} R u^{\prime \prime \prime}\left(y_{1}+s^{*} R\right)\right]
\end{aligned}
$$

from where it follows

$$
\begin{aligned}
& V_{5}^{\prime}\left(s^{*}\right) \approx \frac{\operatorname{Var}_{f}(A)}{2} R u^{\prime \prime \prime}\left(y_{1}+s^{*} R\right) \\
& \quad+\frac{\operatorname{Var}(\tilde{R})}{2} s^{*}\left[2 u^{\prime \prime}\left(y_{1}+s^{*} R\right)+s^{*} R u^{\prime \prime \prime}\left(y_{1}+s^{*} R\right)\right]
\end{aligned}
$$

By Proposition 5.1, $V_{5}^{\prime}(s)$ is a strictly decreasing function, thus $s_{5}^{*}>s^{*}$ iff $0=V_{5}^{\prime}\left(s_{5}^{*}\right)<V_{5}^{\prime}\left(s^{*}\right)$. Taking into account the last approximation of $V_{5}^{\prime}\left(s^{*}\right)$, it follows that $s_{5}^{*}>s^{*}$ iff the following inequality holds:

$$
\begin{aligned}
& \operatorname{Var}_{f}(A) R u^{\prime \prime \prime}\left(y_{1}+s^{*} R\right)+\operatorname{Var}(\tilde{R}) s^{*}\left[2 u^{\prime \prime}\left(y_{1}+s^{*} R\right)\right. \\
& \left.\quad+s^{*} R u^{\prime \prime \prime}\left(y_{1}+s^{*} R\right)\right]>0 .
\end{aligned}
$$

This inequality can be written:

$$
\begin{aligned}
& R u^{\prime \prime \prime}\left(y_{1}+s^{*} R\right)\left[\operatorname{Var}_{f}(A)+\left(s^{*}\right)^{2} \operatorname{Var}(\tilde{R})\right] \\
& +2 s^{*} u^{\prime \prime \prime}\left(y_{1}+s^{*} R\right) \operatorname{Var}(\tilde{R})>0 .
\end{aligned}
$$

Since $u^{\prime \prime}\left(y_{0}+s^{*} R\right)<0$ and $\operatorname{Var}_{f}(A)+\left(s^{*}\right)^{2} \operatorname{Var}(\tilde{R})>$ 0 , the last inequality is equivalent with:

$$
\frac{R s^{*} u^{\prime \prime \prime}\left(y_{1}+s^{*} R\right)}{u^{\prime \prime}\left(y_{1}+s^{*} R\right)}+2 \frac{\left(s^{*}\right)^{2} \operatorname{Var}(\tilde{R})}{\operatorname{Var}_{f}(A)+\left(s^{*}\right)^{2} \operatorname{Var}(\tilde{R})}<0 .
$$

Taking into account (6.1), (6.2) and (6.7), it follows that $s_{5}^{*}>s^{*}$ if and only if $-P R P\left(y_{1}, s^{*} R\right)+K_{1}<0$, from where the equivalence in the statement of theorem is obtained.

Theorem $6.2 s_{6}^{*}>s^{*}$ iff $K_{2}<P R P\left(y_{1}, s^{*} R\right)$

Proof In this proof, we will also use the function $v(y, x)=$ $x u^{\prime}\left(y+s^{*} x\right)$ defined by (6.4). According to (5.7)

$$
\begin{aligned}
V_{6}^{\prime}\left(s^{*}\right) & =-u^{\prime}\left(y_{0}-s^{*}\right)+E_{f}\left[w\left(\tilde{y}, B, s^{*}\right)\right] \\
& =-u^{\prime}\left(y_{0}-s^{*}\right)+E_{f}[v(\tilde{y}, B)]
\end{aligned}
$$

from where, using the first-order condition (4.3), we will have

$V_{6}^{\prime}\left(s^{*}\right)=-R u^{\prime}\left(y_{1}+s^{*}\right)+E_{f}[v(\tilde{y}, B)]$

By applying Proposition 3.3, we can apply the approximation formula:

$$
\begin{aligned}
& E_{f}[v(\tilde{y}, B)] \approx v\left(M(\tilde{y}), E_{f}(B)\right)+\frac{1}{2} v_{11}(M(\tilde{y}), \\
& \left.\quad E_{f}(B)\right) \operatorname{Var}(\tilde{y}) \\
& +\frac{1}{2} v_{22}\left(M(\tilde{y}), E_{f}(B)\right) \operatorname{Var}_{f}(B)
\end{aligned}
$$

Since $M(\tilde{y})=y_{1}$ and $E_{f}(B)=R$, the approximation formula becomes:

$$
\begin{aligned}
E_{f}[v(\tilde{y}, B)] \approx & v\left(y_{1}, R\right)+\frac{1}{2} v_{11}\left(y_{1}, R\right) \operatorname{Var}(\tilde{y}) \\
& +\frac{1}{2} v_{22}\left(y_{1}, R\right) \operatorname{Var}_{f}(B)
\end{aligned}
$$

Using the expressions of $v_{11}(y, x)$ and $v_{22}(y, x)$ from the proof of Theorem 6.1, formula (6.9) gets the form:

$$
\begin{aligned}
& E_{f}[v(\tilde{y}, B)] \approx v\left(y_{1}, R\right)+\frac{\operatorname{Var}(\tilde{y})}{2} R u^{\prime \prime \prime}\left(y_{1}+s^{*} R\right) \\
& +\frac{\operatorname{Var}_{f}(B)}{2} s^{*}\left[2 u^{\prime \prime}\left(y_{1}+s^{*} R\right)+s^{*} R u^{\prime \prime \prime}\left(y_{1}+s^{*} R\right)\right]
\end{aligned}
$$

Replacing in (6.8) $E_{f}[v(\tilde{y}, R)]$ with its approximate value from above, it follows

$$
\begin{aligned}
& V_{6}^{\prime}\left(s^{*}\right) \approx \frac{\operatorname{Var}(\tilde{y})}{2} R u^{\prime \prime \prime}\left(y_{1}+s^{*} R\right) \\
& \quad+\frac{\operatorname{Var}_{f}(B)}{2} s^{*}\left[2 u^{\prime \prime}\left(y_{1}+s^{*} R\right)+s^{*} R u^{\prime \prime \prime}\left(y_{1}+s^{*} R\right)\right]
\end{aligned}
$$


Using arguments similar to the ones from the proof of Theorem 6.1, one obtains the following equivalences:

$$
\begin{aligned}
s_{6}^{*}> & s^{*} \text { iff } V_{6}^{\prime}\left(s^{*}\right)>0 \\
& \text { iff } \frac{R s^{*} u^{\prime \prime \prime}\left(y_{1}+s^{*} R\right)}{u^{\prime \prime}\left(y_{1}+s^{*} R\right)}+2 \frac{\left(s^{*}\right)^{2} \operatorname{Var}_{f}(B)}{\operatorname{Var}(\tilde{y})+\left(s^{*}\right)^{2} \operatorname{Var}_{f}(B)}<0 \\
& \text { iff }-P R P\left(y_{1}, s^{*} R\right)+K_{2}<0
\end{aligned}
$$

Remark 6.3 Theorems 6.1 and 6.2 establish necessary and sufficient conditions for the presence of mixed risk $(A, \tilde{R})$ (resp. $(\tilde{y}, B))$ to generate extra-saving.

Example 6.4 Assume that the consumer's utility function $u$ is CRRA-type (Eeckhoudt et al. 2005, p.21): for all $w>0$, $u(w)$ is defined by:

$u(w)= \begin{cases}\frac{w^{1-\gamma}}{1-\gamma} & \gamma \geq 0, \gamma \neq 1 \\ \ln (w) & \gamma=1\end{cases}$

We compute the partial relative prudence index $P R P(y, x)$ associated with $u$.

(a) Case $\gamma \geq 0, \gamma \neq 1$ One notices that

$$
\begin{aligned}
u^{\prime}(x) & =\frac{1}{x^{\gamma}} ; u^{\prime \prime}(x)=-\gamma \frac{1}{x^{\gamma+1}} ; u^{\prime \prime \prime}(x) \\
& =\gamma(\gamma+1) \frac{1}{x^{\gamma+2}}
\end{aligned}
$$

from where it follows

$$
P R P(y, x)=(\gamma+1) \frac{x}{y+x}
$$

(b) Case $\gamma=1$. An analogous computation leads to

$$
P R P(y, x)=2 \frac{x}{y+x}
$$

We will compute the indicators $K_{1}, K_{2}$ under the following hypotheses:

- the weighting function $f$ has the form $f(t)=2 t$, for $t \in[0,1]$;

- the random variables $\tilde{y}$ and $\tilde{R}$ follow uniform distributions on the interval $[c, d]$, where $0<c<d$;

- the fuzzy numbers $A$ and $B$ are defined by $a_{1}(t)=$ $b_{1}(t)=c$ and $a_{2}(t)=b_{2}(t)=d$, for any $t \in[0,1]$.
By a simple calculation, we find the following possibilistic and probabilistic variances:

$$
\begin{gathered}
\operatorname{Var}_{f}(A)=\operatorname{Var}_{f}(B)=\frac{(c-d)^{2}}{4} \\
\operatorname{Var}(\tilde{y})=\operatorname{Var}(\tilde{R})=\frac{(c-d)^{2}}{12}
\end{gathered}
$$

Replacing those variances in formulas (6.2) and (6.3), one obtains

$K_{1}=2 \frac{\left(s^{*}\right)^{2}}{3+\left(s^{*}\right)^{2}} ; K_{2}=2 \frac{\left(s^{*}\right)^{2}}{1+3\left(s^{*}\right)^{2}}$

Taking into account (6.11)-(6.13) by applying Theorems 6.1 and 6.2, the necessary and sufficient conditions on extrasaving get the form of the following equivalence:

- Case (a):

$$
\begin{aligned}
s_{5}^{*}-s^{*}>0 & \text { iff } \frac{2 s^{*}}{3+\left(s^{*}\right)^{2}}<\frac{(\gamma+1) R}{y_{1}+s^{*} R} \\
s_{6}^{*}-s^{*}>0 & \text { iff } \frac{2 s^{*}}{1+3\left(s^{*}\right)^{2}}<\frac{(\gamma+1) R}{y_{1}+s^{*} R}
\end{aligned}
$$

- Case (b):

$$
\begin{aligned}
& s_{5}^{*}-s^{*}>0 \text { iff } s^{*}<3 \frac{R}{y_{1}} ; \\
& s_{6}^{*}-s^{*}>0 \text { iff } 2 R\left(s^{*}\right)^{2}-y_{1} s^{*}+R>0 .
\end{aligned}
$$

The extra-savings from Theorems 6.1 and 6.2 are formulated according to the indicators $K_{1}, K_{2}$ and $R P R\left(y_{1}, s^{*} R\right)$, which in their turn depend on $s^{*}$, the optimal saving for the certain model (4.2). By the first-order condition (4.3), we have the following equation in $s^{*}$ :

$-u^{\prime}\left(y_{0}-s^{*}\right)+R u^{\prime}\left(y_{1}+s^{*} R\right)=0$

Finding the exact solution of Eq. (6.14) is difficult; therefore, we search for an approximate value of $s^{*}$. Considering the Taylor approximation

$$
\begin{gathered}
u^{\prime}\left(y_{0}-s^{*}\right) \approx u^{\prime}\left(y_{0}\right)-s^{*} u^{\prime \prime}\left(y_{0}\right) ; u^{\prime}\left(y_{1}+s^{*} R\right) \approx u^{\prime}\left(y_{1}\right) \\
+s^{*} R u^{\prime \prime}\left(y_{1}\right)
\end{gathered}
$$

and replacing in (6.14), one obtains the following approximation formula for $s^{*}$ :

$s^{*} \approx \frac{u^{\prime}\left(y_{0}\right)-R u^{\prime}\left(y_{1}\right)}{u^{\prime \prime}\left(y_{0}\right)+R^{2} u^{\prime \prime}\left(y_{1}\right)}$ 
Replacing $s^{*}$ in (6.2) and (6.3) with its approximate value from (6.15), it follows

$$
\begin{aligned}
& K_{1}=2\left[\frac{u^{\prime}\left(y_{0}\right)-R u^{\prime}\left(y_{1}\right)}{u^{\prime \prime}\left(y_{0}\right)+R^{2} u^{\prime \prime}\left(y_{1}\right)}\right]^{2} \\
& \frac{\operatorname{Var}(\tilde{R})}{\operatorname{Var}_{f}(A)+\left[\frac{u^{\prime}\left(y_{0}\right)-R u^{\prime}\left(y_{1}\right)}{u^{\prime \prime}\left(y_{0}\right)+R^{2} u^{\prime \prime}\left(y_{1}\right)}\right]^{2} \operatorname{Var}(\tilde{R})} \\
& K_{2}=2\left[\frac{u^{\prime}\left(y_{0}\right)-R u^{\prime}\left(y_{1}\right)}{u^{\prime \prime}\left(y_{0}\right)+R^{2} u^{\prime \prime}\left(y_{1}\right)}\right]^{2} \\
& \operatorname{Var}_{f}(\tilde{y})+\left[\frac{u^{\prime}\left(y_{0}\right)-R u^{\prime}\left(y_{1}\right)}{u^{\prime \prime}\left(y_{0}\right)+R^{2} u^{\prime \prime}\left(y_{1}\right)}\right]^{2} \operatorname{Var}_{f}(B)
\end{aligned}
$$

Also, by (6.1) and (6.13) one can compute an approximate value of the partial relative prudence index:

$$
\begin{aligned}
& P R P\left(y_{1}, s^{*} R\right)=-R s^{*} \frac{u^{\prime \prime \prime}\left(y_{1}+s^{*} R\right)}{u^{\prime \prime}\left(y_{1}+s^{*} R\right)} \\
& \approx R \frac{u^{\prime}\left(y_{0}\right)-R u^{\prime}\left(y_{1}\right)}{u^{\prime \prime}\left(y_{0}\right)+R^{2} u^{\prime \prime}\left(y_{1}\right)} \frac{u^{\prime \prime \prime}\left(y_{1}+R \frac{u^{\prime}\left(y_{0}\right)-R u^{\prime}\left(y_{1}\right)}{u^{\prime \prime}\left(y_{0}\right)+R^{2} u^{\prime \prime}\left(y_{1}\right)}\right)}{u^{\prime \prime}\left(y_{1}+R \frac{u^{\prime}\left(y_{0}\right)-R u^{\prime}\left(y_{1}\right)}{u^{\prime \prime}\left(y_{0}\right)+R^{2} u^{\prime \prime}\left(y_{1}\right)}\right)}
\end{aligned}
$$

Using the approximate values of $K_{1}, K_{2}$ and $P R P\left(y_{1}, s^{*} R\right)$ from (6.16)-(6.18), we can verify the extra-saving conditions from Theorems 6.1 and 6.2 in different concrete cases.

Example 6.5 Let $c, d \in \mathbf{R}$ with $0<c<d$. We will analyze the mixed saving model (5.4) under the following hypotheses:

- the weighting function is $f(\gamma)=2 \gamma, \gamma \in[0,1]$;

- The utility function of the consumer is $u(x)=-e^{-x}$, $x \in \mathbf{R}$;

- the labor income risk is the fuzzy number $A$ defined by $a_{1}(\gamma)=c, a_{2}(\gamma)=d$ for any $\gamma \in[0,1]$;

- the return of saving is the random variable $\tilde{R}$ which follows the uniform distribution on the interval $[c, d]$;

- $y_{0}=y_{1}$ (we recall that $y_{0}$ is the sure income from period $0, y_{1}=E_{f}(A)$ and $\left.R=M(\tilde{R})\right)$

A simple calculation shows that

$$
\begin{gathered}
E_{f}(A)=M(\tilde{R})=\frac{c+d}{2} ; \operatorname{Var}_{f}(A)=\frac{(c-d)^{2}}{4} ; \\
\operatorname{Var}(\tilde{R})=\frac{(c-d)^{2}}{12}
\end{gathered}
$$

One notices that $u^{\prime}(x)=u^{\prime \prime \prime}(x)=e^{-x}$ and $u^{\prime \prime}(x)=-e^{-x}$. Applying (6.15), one obtains the following approximate value of the optimal saving $s^{*}$ :

$$
\begin{aligned}
s^{*} & \approx \frac{u^{\prime}\left(y_{0}\right)-R u^{\prime}\left(y_{1}\right)}{u^{\prime \prime}\left(y_{0}\right)+R^{2} u^{\prime \prime}\left(y_{1}\right)}=\frac{e^{-y_{0}}-R e^{-y_{1}}}{-e^{-y_{0}}-R^{2} e^{-y_{1}}} \\
& =\frac{R-1}{R^{2}+1}
\end{aligned}
$$

Exactly as in Example 6.4, by using the two variances from (6.19) one reaches $K_{1}=2 \frac{\left(s^{*}\right)^{2}}{3+\left(s^{*}\right)^{2}}$. Replacing $s^{*}$ in this expression of $K_{1}$ with its approximate value (6.20), it follows:

$$
\begin{aligned}
K_{1} & =\frac{2}{\frac{3}{\left(s^{*}\right)^{2}}+1}=\frac{2}{3\left(\frac{R^{2}+1}{R-1}\right)^{2}+1} \\
& =\frac{2(R-1)^{2}}{3\left(R^{2}+1\right)^{2}+(R-1)^{2}}
\end{aligned}
$$

For the utility function $u(x)=-e^{-x}$, we have $P R P(y, x)$ $=x$, and thus, using (6.18), one obtains

$P R P\left(y_{1}, s^{*} R\right)=R s^{*} \approx \frac{R(R-1)}{R^{2}+1}$

Taking into account (6.21) and (6.22), the equivalence of Theorem 6.1 becomes:

$$
\begin{aligned}
& s_{5}^{*}-s^{*}>0 \text { iff } \frac{2(R-1)^{2}}{3\left(R^{2}+1\right)^{2}+(R-1)^{2}}<\frac{R(R-1)}{R^{2}+1} \\
& \quad \text { iff } 2(R-1)^{2}\left(R^{2}+1\right)<R(R-1)\left[3\left(R^{2}+1\right)^{2}+(R-1)^{2}\right]
\end{aligned}
$$

If $R>1$, then

$$
\begin{aligned}
& s_{5}^{*}-s^{*}>0 \\
& \quad \text { iff } 2(R-1)\left(R^{2}+1\right)<R\left[3\left(R^{2}+1\right)^{2}+(R-1)^{2}\right]
\end{aligned}
$$

and if $R<1$ then

$$
\begin{aligned}
& s_{5}^{*}-s^{*}>0 \\
& \quad \text { iff } 2(R-1)\left(R^{2}+1\right)>R\left[3\left(R^{2}+1\right)^{2}+(R-1)^{2}\right] .
\end{aligned}
$$

\section{Generalized precautionary saving}

Usually the precautionary saving shows the change in optimal saving when moving from a certain model to a model with risk. The notion of precautionary saving can be extended in the following way: if $\tilde{s}_{1}, \tilde{s}_{2}$ are the optimal saving levels for $M_{1}, M_{2}$ then $\tilde{s}_{1}-\tilde{s}_{2}$ is the precautionary saving corresponding to moving from $M_{1}$ to $M_{2}$. Of course the notion of precautionary saving thus defined has a meaning when the model $M_{2}$ is obtained from $M_{1}$ by adding one or more risk parameters. Such a notion has been studied in Courbage and 
Rey (2007) and Magnani and Menegatti (2015) for probabilistic models and in Georgescu (2014) and Georgescu and Kinnunen (2016) for possibilistic models.

In this section, we will consider four notions of precautionary saving (in generalized sense) which reflect the changes in optimal saving in the framework of the mixed models in Sect. 5. We will notice that the mixed models $(A, \tilde{R})$ and $(\tilde{y}, B)$ from Sect. 5 can be obtained from models with a single type of risk $(\tilde{y}, R),\left(y_{1}, \tilde{R}\right),(A, R)$ and $\left(y_{1}, B\right)$ from Sect. 4 by adding another type of risk, according to the following routes:

$$
\begin{aligned}
& \left(y_{1}, \tilde{R}\right) \rightarrow(A, \tilde{R}) ;(A, R) \rightarrow(A, \tilde{R}) \\
& \left(y_{1}, B\right) \rightarrow(\tilde{y}, B) ;(\tilde{y}, R) \rightarrow(\tilde{y}, B) .
\end{aligned}
$$

The changes in optimal saving generated by these transformations will be measured by the following precautionary savings:

- $s_{5}^{*}-s_{2}^{*}$ : on the route $\left(y_{1}, \tilde{R}\right) \rightarrow(A, \tilde{R})$;

- $s_{5}^{*}-s_{3}^{*}$ : on the route $(A, R) \rightarrow(A, \tilde{R})$;

- $s_{6}^{*}-s_{4}^{*}$ : on the route $\left(y_{1}, B\right) \rightarrow(\tilde{y}, B)$;

- $s_{5}^{*}-s_{1}^{*}$ : on the route $(\tilde{y}, R) \rightarrow(\tilde{y}, B)$.

For instance, $s_{5}^{*}-s_{2}^{*}$ indicated the change in optimal saving when moving from the model $\left(y_{1}, \tilde{R}\right)$ to the mixed model $(A, \tilde{R})$, transforming the certain value $y_{1}$ into the fuzzy number $A$.

We will establish next necessary and sufficient conditions for the four transformations from above to generate extrasaving. We fix a weighting function $f:[0,1] \rightarrow \mathbf{R}$, and we keep the notations from the previous sections.

Theorem $7.1 s_{5}^{*}-s_{2}^{*}>0$ if and only if $u^{\prime \prime \prime}\left(y_{1}+s_{2}^{*} R\right)>0$.

Proof By Proposition 5.1, $V_{5}^{\prime}(s)$ is a strictly decreasing function, thus

$s_{5}^{*}-s_{2}^{*}>0$ iff $V_{5}^{\prime}\left(s_{5}^{*}\right)<V_{5}^{\prime}\left(s_{2}^{*}\right)$

Intending to find an approximation of $V_{5}^{\prime}\left(s_{5}^{*}\right)$, we will apply first (5.3):

$V_{5}^{\prime}\left(s_{2}^{*}\right)=-u^{\prime}\left(y_{0}-s_{2}^{*}\right)+E_{f}\left[w\left(A, \tilde{R}, s_{2}^{*}\right)\right]$

where $w(y, x, s)=x u^{\prime}(y+s x)$, by (5.2). Introducing the bidimensional function

$g(y, x)=w\left(y, x, s_{2}^{*}\right)=x u^{\prime}\left(y+s_{2}^{*} x\right)$

and taking into account the first-order condition (4.7), formula (7.2) becomes

$$
V_{5}^{\prime}\left(s_{2}^{*}\right)=-M\left[g\left(y_{1}, \tilde{R}\right)\right]+E_{f}[g(A, \tilde{R})]
$$

We are looking for approximations of the two terms from (7.4). Exactly as in (6.6) (with $g$ instead of $v$ ), the following approximation holds:

$$
\begin{aligned}
& E_{f}[g(A, \tilde{R})] \approx g\left(y_{1}, R\right)+\frac{1}{2} g_{11}\left(y_{1}, R\right) \operatorname{Var}_{f}(A) \\
& \quad+\frac{1}{2} g_{22}\left(y_{1}, R\right) \operatorname{Var}(\tilde{R})
\end{aligned}
$$

Considering the following function

$h(x)=w\left(y_{1}, x, s_{2}^{*}\right)=g\left(y_{1}, x\right)=x u^{\prime}\left(y_{1}+s_{2}^{*} x\right)$

one will have $M\left[g\left(y_{1}, \tilde{R}\right)\right]=E_{f}[h(\tilde{R})]$ and

$h^{\prime \prime}(x)=2 s_{2}^{*} u^{\prime \prime}\left(y_{1}+s_{2}^{*} x\right)+\left(s_{2}^{*}\right)^{2} x u^{\prime \prime \prime}\left(y_{1}+s_{2}^{*} x\right)$

For the probabilistic expected utility $M[h(\tilde{R})]$, one will use the known approximation (by Eeckhoudt et al. 2005 and Gollier 2004):

$$
\begin{aligned}
& M[h(\tilde{R})] \approx h(M(\tilde{R})) \\
& \quad+\frac{h^{\prime \prime}(M(\tilde{R}))}{2} \operatorname{Var}(\tilde{R})=h(R)+\frac{h^{\prime \prime}(R)}{2} \operatorname{Var}(\tilde{R})
\end{aligned}
$$

Exactly as in the proof of Theorem 6.1 (with $g$ instead of $v$ ), one will have

$$
\begin{aligned}
& g_{11}\left(y_{1}, R\right)=R u^{\prime \prime \prime}\left(y_{1}+s_{2}^{*} R\right) \\
& g_{22}\left(y_{1}, R\right)=2 s_{2}^{*} u^{\prime \prime}\left(y_{1}+s_{2}^{*} R\right)+\left(s_{2}^{*}\right)^{2} R u^{\prime \prime \prime}\left(y_{1}+s_{2}^{*} R\right)
\end{aligned}
$$

From (7.7) and (7.10), it follows $h^{\prime \prime}(R)=g_{22}\left(y_{1}, R\right)$. Also, $h(R)=g\left(y_{1}, R\right)$; thus, by (7.4), (7.5) and (7.6), $V_{5}^{\prime}\left(s_{2}^{*}\right)$ can be approximated as such:

$V_{5}^{\prime}\left(s_{2}^{*}\right) \approx \frac{1}{2} g_{11}\left(y_{1}, R\right) \operatorname{Var}_{f}(A)$

Taking into account that $\operatorname{Var}_{f}(A)>0$, from (7.1) and (7.11) it follows that $s_{5}^{*}>s_{2}^{*}$ iff $g_{11}\left(y_{1}, R\right)>0$. Considering further (7.9) and $R>0$ the following equivalence is obtained: $s_{5}^{*}-s_{2}^{*}>0$ iff $u^{\prime \prime \prime}\left(y_{1}+s_{2}^{*} R\right)>0$.

Theorem $7.2 s_{5}^{*}-s_{3}^{*}>0$ iff $P R P\left(y_{1}, s_{3}^{*} R\right)>2$

Proof As in the proof of Theorem 7.1, the following equivalence holds:

$s_{5}^{*}>s_{3}^{*}>0$ iff $V_{5}^{\prime}\left(s_{3}^{*}\right)>0$

Introducing the function

$h(y, x)=w\left(y, x, s_{3}^{*}\right)$ 
it follows

$$
\begin{aligned}
V_{5}^{\prime}\left(s_{3}^{*}\right) & =-u^{\prime}\left(y_{0}-s_{3}^{*}\right)+E_{f}\left[w\left(A, \tilde{R}, s_{3}^{*}\right)\right] \\
& =-u^{\prime}\left(y_{1}-s_{3}^{*}\right)+E_{f}[k(A, \tilde{R})]
\end{aligned}
$$

Taking into account the first-order condition (4.9), we have $u^{\prime}\left(y_{0}-s_{3}^{*}\right)=E_{f}\left[R u^{\prime}\left(A+R s_{3}^{*}\right)\right]$ thus

$V_{5}^{\prime}\left(s_{3}^{*}\right)=-E_{f}\left[R u^{\prime}\left(A+R s_{3}^{*}\right)\right]+E_{f}[k(A, \tilde{R})]$

Applying Proposition 3.3, we approximate $E_{f}[k(A, \tilde{R})]$ :

$$
\begin{gathered}
E_{f}[k(A, \tilde{R})] \approx \\
k\left(y_{1}, R\right)+\frac{1}{2} k_{11}\left(y_{1}, R\right) \operatorname{Var}_{f}(A) \\
+\frac{1}{2} k_{22}\left(y_{1}, R\right) \operatorname{Var}(\tilde{R})
\end{gathered}
$$

We consider the following function:

$l(y)=w\left(y, R, s_{3}^{*}\right)=k(y, R)=R u^{\prime}\left(y+s_{3}^{*} R\right)$

As in the proof of Proposition 7.1, one has

$$
\begin{aligned}
& l\left(y_{1}\right)=h\left(y_{1}, R\right)=R u^{\prime}\left(y_{1}+s_{3}^{*} R\right) \\
& k_{11}\left(y_{1}, R\right)=l^{\prime \prime}\left(y_{1}\right)=R u^{\prime \prime \prime}\left(y_{1}+s_{3}^{*} R\right) \\
& k_{12}\left(y_{1}, R\right)=s_{3}^{*}\left[2 u^{\prime \prime}\left(y_{1}+s_{3}^{*} R\right)+s_{3}^{*} R u^{\prime \prime \prime}\left(y_{1}+s_{3}^{*} R\right)\right]
\end{aligned}
$$

Noticing that $E_{f}\left[R u^{\prime}\left(A+R s_{3}^{*}\right)\right]=E_{f}[l(A)]$ and applying Proposition 2.4, one obtains the approximation:

$E_{f}\left[R u^{\prime}\left(A+R s_{3}^{*}\right)\right] \approx l\left(y_{1}\right)+\frac{l^{\prime \prime}\left(y_{1}\right)}{2} \operatorname{Var}_{f}(A)$

From formulas (7.14) and (7.15) and (7.17)-(7.20), it follows

$$
\begin{aligned}
& V_{5}^{\prime}\left(s_{3}^{*}\right) \approx \frac{1}{2} k_{22}\left(y_{1}, R\right) \operatorname{Var}(\tilde{R}) \\
& \quad=\frac{\operatorname{Var}(\tilde{R})}{2} s_{3}^{*}\left[2 u^{\prime \prime}\left(y_{1}+s_{3}^{*} R\right)+s_{3}^{*} R u^{\prime \prime \prime}\left(y_{1}+s_{3}^{*} R\right)\right]
\end{aligned}
$$

Taking into account the previous relation and (7.12), the following equivalence holds:

$$
s_{5}^{*}>s_{3}^{*} \text { iff } 2 u^{\prime \prime}\left(y_{1}+s_{3}^{*} R\right)+s_{3}^{*} R u^{\prime \prime \prime}\left(y_{1}+s_{3}^{*} R\right)>0
$$

Since $u^{\prime \prime}\left(y_{1}+s_{3}^{*} R\right)<0$ from (7.21), it follows immediately that $s_{5}^{*}>s_{3}^{*}$ iff $\operatorname{PRP}\left(y_{1}, s_{3}^{*} R\right)>2$.

Analogously to Theorem 7.1 and 7.2, the following results can be proved:
Theorem 7.3 $s_{6}^{*}-s_{4}^{*}>0$ if and only if $u^{\prime \prime \prime}\left(y_{1}+s_{4}^{*} R\right)>0$.

Theorem 7.4 $s_{5}^{*}-s_{1}^{*}>0$ if and only if $P R P\left(y_{1}, s_{1}^{*} R\right)>2$.

Theorems 7.1 and 7.3 belong to the research direction from papers Leland (1968), Sandmo (1970), Drèze and Modigliani (1972), Courbage and Rey (2007) and Lucia-Casademunt and Georgescu (2013): the generation of extra-saving by adding a (probabilistic or possibilistic) labor income risk is characterized by a condition of prudence of the consumer (by Kimball 1990). The other two results (Theorem 7.2 and 7.4) will be registered in the research stream from Sandmo (1970), Rothschild and Stiglitz (1971), Eeckhoudt et al. (2005) and Magnani (2017): the generation of extra-saving by adding a (probabilistic or possibilistic) interest-rate risk holds if and only if the (partial) relative prudence index is greater than 2 .

Example 7.5 We analyze the conditions of extra-saving from the four theorems of the sections under the hypothesis from Example 6.4. First we notice that $u^{\prime \prime \prime}(x)=\gamma(\gamma+1) \frac{1}{x^{\gamma+2}}>0$ for any $x>0$; thus, the prudence conditions of the consumer from Theorems 7.1 and 7.3 are fulfilled. Accordingly, $s_{5}^{*}-$ $s_{2}^{*}>0$ and $s_{6}^{*}-s_{4}^{*}>0$; in both cases, the presence of a (probabilistic and possibilistic) labor income risk generates extra-saving.

From (6.12) and (6.13), the following formula of the partial relative prudence index is deduced:

$\operatorname{PRP}\left(y_{1}, s_{3}^{*} R\right)=\left\{\begin{array}{lc}\frac{(\gamma+1) s_{3}^{*} R}{y_{1}+s_{3}^{*} R} \\ 2 \frac{s_{3}^{*} R}{y_{1}+s_{3}^{*} R} \text { if } \quad \text { if } \quad \gamma \neq 0, \gamma \neq 1\end{array}\right.$

Then, by applying Theorem 7.2 the following necessary and sufficient conditions of extra-saving will be obtained:

- for $\gamma>1: s_{5}^{*}-s_{3}^{*}>0$ iff $\frac{(\gamma+1) s_{3}^{*} R}{y_{1}+s_{3}^{*} R}>2$ iff $s_{3}^{*}>\frac{2 y_{1}}{(\gamma-1) R}$;

- for $\gamma<1: s_{5}^{*}-s_{3}^{*}>0$ iff $\frac{(\gamma+1) s_{3}^{*} R}{y_{1}+s_{3}^{*} R}>2$ iff $s_{3}^{*}<\frac{2 y_{1}}{(\gamma-1) R}$;

- for $\gamma=1: s_{5}^{*}-s_{3}^{*}>0$ iff $2 \frac{s_{3}^{*} R}{y_{1}+s_{3}^{*} R}>2$ iff $s_{3}^{*}>\frac{y_{1}}{R}$;

The necessary and sufficient conditions of extra-saving from Theorem 7.4 are treated similarly.

\section{Approximation of optimal saving}

This section is concerned with the computation of the optimal savings $s_{5}^{*}$ and $s_{6}^{*}$ corresponding to the mixed models (5.4) and (5.8). According to the first-order conditions (5.5) and (5.9), $s_{5}^{*}$ and $s_{6}^{*}$ verify the following equations:

$$
\begin{aligned}
& -u^{\prime}\left(y_{0}-s_{5}^{*}\right)+E_{f}\left[w\left(A, \tilde{R}, s_{5}^{*}\right)\right]=0 \\
& -u^{\prime}\left(y_{0}-s_{6}^{*}\right)+E_{f}\left[w\left(A, \tilde{R}, s_{6}^{*}\right)\right]=0
\end{aligned}
$$


where $w(y, x, s)=x u^{\prime}(y+s x)$. Since finding the exact solution of their equations is difficult, we will present in this section a way to obtain their approximate solutions.

We will assume that the utility function $u$ is of class $\mathcal{C}^{4}$. One will deal first with Eq. (8.1). We introduce the following notations:

$C_{0}=-u^{\prime}\left(y_{0}\right)+R\left[u^{\prime}\left(y_{1}\right)+\frac{1}{2} \operatorname{Var}_{f}(A) u^{\prime \prime \prime}\left(y_{1}\right)\right]$

$C_{1}=u^{\prime \prime}\left(y_{0}\right)+\left(R^{2}+\operatorname{Var}(\tilde{R})\right) u^{\prime \prime}\left(y_{1}\right)+\frac{R^{2}}{2} \operatorname{Var}_{f}(A) u^{i v}\left(y_{1}\right)$

$C_{2}=\frac{3 R}{2} \operatorname{Var}(\tilde{R}) u^{\prime \prime \prime}\left(y_{1}\right)$

$C_{3}=\frac{R^{2}}{2} \operatorname{Var}(\tilde{R}) u^{i v}\left(y_{1}\right)$

Theorem 8.1 An approximate value of the optimal saving $s_{5}^{*}$ can be computed as a solution of the third-order equation in $z:$

$C_{3} z^{3}+C_{2} z^{2}+C_{1} z+C_{0}=0$

Proof Introducing the following function

$\theta(y, x)=w\left(y, x, s_{5}^{*}\right)=x u^{\prime}\left(y+s_{5}^{*} x\right)$

Eq. (8.1) gets the form:

$-u^{\prime}\left(y_{0}-s_{5}^{*}\right)+E_{f}[\theta(A, \tilde{R})]=0$

We apply Proposition 3.3 for the mixed vector $(A, \tilde{R})$ and for the bidimensional utility function $\theta(y, x)$ of class $\mathcal{C}^{2}$ :

$$
\begin{gathered}
E_{f}[\theta(A, \tilde{R})] \approx \theta\left(E_{f}(A), M(\tilde{R})\right)+\frac{1}{2} \theta_{11}\left(E_{f}(A),\right. \\
M(\tilde{R})) \operatorname{Var}_{f}(A)+\frac{1}{2} \theta_{22}\left(E_{f}(A), M(\tilde{R})\right) \operatorname{Var}(X)
\end{gathered}
$$

Since by the construction of the mixed saving model (5.4) we have $E_{f}(A)=y_{1}$ and $M(\tilde{R})=R$, the previous formula becomes:

$$
\begin{aligned}
E_{f}[\theta(A, \tilde{R})] \approx & \theta\left(y_{1}, R\right)+\frac{1}{2} \theta_{11}\left(y_{1}, R\right) \operatorname{Var}_{f}(A) \\
& +\frac{1}{2} \theta_{22}\left(y_{1}, R\right) \operatorname{Var}(\tilde{R})
\end{aligned}
$$

By a reasoning analogous to the one from the proof of Theorem 6.1 (with $\theta(y, x)$ instead of $v(y, x)$ and with $s_{5}^{*}$ instead of $s^{*}$ ), formula (8.10) will become:

$$
\begin{aligned}
E_{f}[\theta(A, \tilde{R})] \approx & R u^{\prime}\left(y_{1}+s_{5}^{*} R\right)+\frac{\operatorname{Var}_{f}(A)}{2} R u^{\prime \prime}\left(y_{1}+s_{5}^{*} R\right) \\
& +\frac{\operatorname{Var}(\tilde{R})}{2} s_{5}^{*}\left[2 u^{\prime \prime}\left(y_{1}+s_{5}^{*} R\right)+s_{5}^{*} u^{\prime \prime \prime}\left(y_{1}+s_{5}^{*} R\right)\right]
\end{aligned}
$$

Since $u$ is of class $\mathcal{C}^{4}$, one can consider the first-order Taylor approximation:

$$
\begin{gathered}
u^{\prime}\left(y_{0}-s_{5}^{*}\right) \approx u^{\prime}\left(y_{0}\right)-s_{5}^{*} u^{\prime \prime}\left(y_{0}\right) \\
u^{\prime}\left(y_{1}+s_{5}^{*} R\right) \approx u^{\prime}\left(y_{1}\right)+s_{5}^{*} R u^{\prime \prime}\left(y_{1}\right) ; \\
u^{\prime \prime}\left(y_{1}+s_{5}^{*} R\right) \approx u^{\prime \prime}\left(y_{1}\right)+s_{5}^{*} R u^{\prime \prime \prime}\left(y_{1}\right) \\
u^{\prime \prime \prime}\left(y_{1}+s_{5}^{*} R\right) \approx u^{\prime \prime \prime}\left(y_{1}\right)+s_{5}^{*} R u^{i v}\left(y_{1}\right) .
\end{gathered}
$$

Taking into account these approximations and formula (8.11), Eq. (8.9) becomes:

$$
\begin{gathered}
-u^{\prime}\left(y_{0}\right)+s_{5}^{*} u^{\prime \prime}\left(y_{0}\right)+R\left[u^{\prime}\left(y_{1}\right)+s_{5}^{*} R u^{\prime \prime}\left(y_{1}\right)\right] \\
+\frac{\operatorname{Var}_{f}(A)}{2} R\left[u^{\prime \prime \prime}\left(y_{1}\right)+s_{5}^{*} R u^{i v}\left(y_{1}\right)\right] \\
+\frac{\operatorname{Var}(\tilde{R})}{2} s_{5}^{*}\left[2 \left(u^{\prime \prime}\left(y_{1}\right)+s_{5}^{*} R u^{\prime \prime \prime}\left(y_{1}\right)\right.\right. \\
\left.+s_{5}^{*} R\left(u^{\prime \prime \prime}\left(y_{1}\right)+s_{5}^{*} R u^{i v}\left(y_{1}\right)\right)\right] \approx 0
\end{gathered}
$$

Ordering by the powers of $s_{5}^{*}$, one obtains

$$
\begin{aligned}
& \frac{R^{2}}{2} \operatorname{Var}(\tilde{R}) u^{i v}\left(y_{1}\right)\left(s_{5}^{*}\right)^{3}+\frac{3 R}{2} \operatorname{Var}(\tilde{R}) u^{\prime \prime \prime}\left(y_{1}\right)\left(s_{5}^{*}\right)^{2} \\
& \quad+\left[u^{\prime \prime}\left(y_{0}\right)+\left(R^{2}+\operatorname{Var}(\tilde{R})\right) u^{\prime \prime}\left(y_{1}\right)\right. \\
& \left.\quad+\frac{R^{2}}{2} \operatorname{Var}_{f}(A) u^{i v}\right] s_{5}^{*} \\
& \quad-u^{\prime}\left(y_{0}\right)+R\left[u^{\prime}\left(y_{1}\right)+\frac{1}{2} \operatorname{Var}_{f}(A) u^{\prime \prime \prime}\left(y_{1}\right)\right] \approx 0 .
\end{aligned}
$$

The coefficients of this equation are exactly $C_{0}, C_{1}, C_{2}$ and $C_{3}$ from formulas (8.3)-(8.6); thus, $s_{5}^{*}$ has as approximate solution Eq. (8.7).

Remark 8.2 According to the previous theorem, an approximate value of the optimal saving $s_{5}^{*}$ is determined as a solution of the third-order equation (8.7). From Remark 5.3, one knows that $s_{5}^{*}$ is the unique solution of (8.1) (when it exists). Accordingly, the third-order equation (8.7) should have a unique real solution. This is done when the discriminant of this equation:

$\delta=18 C_{3} C_{2} C_{1} C_{0}-4 C_{2}^{3} C_{0}+C_{2}^{2} C_{1}^{2}-4 C_{3} C_{1}^{3}-27 C_{3}^{2} C_{0}^{2}$

is strictly negative. The solution $s_{5}^{*}$ of Eq. (8.7) will be obtained applying the Cardano formulas to solve third-order algebraic equations. 
To determine an approximate solution of Eq. (8.2), one will proceed similarly.

$$
\begin{aligned}
& D_{0}=-u^{\prime}\left(y_{0}\right)+R\left[u^{\prime}\left(y_{1}\right)+\frac{1}{2} \operatorname{Var}(\tilde{y}) u^{\prime \prime \prime}\left(y_{1}\right)\right] \\
& D_{1}=u^{\prime \prime}\left(y_{0}\right)+\left(R^{2}+\operatorname{Var}_{f}(B)\right) u^{\prime \prime}\left(y_{1}\right)+\frac{R^{2}}{2} \operatorname{Var}(\tilde{y}) u^{i v}\left(y_{0}\right) \\
& D_{2}=\frac{3 R}{2} \operatorname{Var}(\tilde{R}) u^{\prime \prime \prime}\left(y_{1}\right) \\
& D_{3}=\frac{R^{2}}{2} \operatorname{Var}(\tilde{R}) u^{i v}\left(y_{1}\right)
\end{aligned}
$$

Theorem 8.3 An approximate value of the optimal saving $s_{6}^{*}$ will be the solution of the third-degree equation in $z$ :

$$
D_{3} z^{3}+D_{2} z^{2}+D_{1} z+D_{0}=0
$$

Proof By a reasoning similar to the one in the proof of Theorem 8.1 , replacing the mixed vector $(A, \tilde{R})$ by the mixed vector $(\tilde{y}, B)$.

Example 8.4 Let $c, d \in \mathbf{R}$ such that $0<c<d$. We consider the saving model (5.4) under the hypothesis of Example 6.5; (a) the weighting function is $f(\gamma)=2 \gamma, \gamma \in[0,1]$; (b) the utility function is $u(x)=-e^{-x}$, for all $x \in \mathbf{R}$; (c) the labor income risk is the fuzzy number $A$ defined by $a_{1}(\gamma)=c$ and $a_{2}(\gamma)=d$, for all $\gamma \in[0,1]$; (d) the return of saving is the uniform distribution $\tilde{R}$ on the interval $[c, d]$; (e) $y_{0}=$ $y_{1}=E_{f}(A)=E_{f}(\tilde{R})=R=\frac{c+d}{2}$. One notices that $u^{\prime}(x)=u^{\prime \prime \prime}(x)=e^{-x}$ and $u^{\prime \prime}(x)=u^{i v}(x)=-e^{-x}$ for any $x \in \mathbf{R}$.

We compute the coefficients $C_{0}, C_{1}, C_{2}, C_{3}$ with formulas (8.3)-(8.6):

$$
\begin{aligned}
& C_{0}=e^{-y_{0}}\left[-1+R+\frac{R}{2} \operatorname{Var}_{f}(A)\right] \\
& C_{1}=-e^{-y_{0}}\left[1+R^{2}+\operatorname{Var}(\tilde{R})+\frac{R^{2}}{2} \operatorname{Var}_{f}(A)\right] \\
& C_{2}=e^{-y_{0}} \frac{3 R}{2} \operatorname{Var}(\tilde{R}) \\
& C_{3}=-e^{-y_{0}} \frac{R^{2}}{2} \operatorname{Var}(\tilde{R}) .
\end{aligned}
$$

Replacing in Eq. (8.7) $C_{0}, C_{1}, C_{2}$ and $C_{3}$ with the above values, one obtains the third-order equation:

$$
\begin{aligned}
& -\frac{R^{2}}{2} \operatorname{Var}(\tilde{R}) z^{3}+\frac{3 R}{2} \operatorname{Var}(\tilde{R}) z^{2} \\
& -\left[1+R^{2}+\operatorname{Var}(\tilde{R})+\frac{R^{2}}{2} \operatorname{Var}_{f}(A)\right] z-1+R \\
& +\frac{R}{2} \operatorname{Var}_{f}(A)=0
\end{aligned}
$$

Let's take the numerical example $c=4, d=6$. Then, by $(6,17)$ one has $R=M(\tilde{R})=\frac{c+d}{2}=5, \operatorname{Var}_{f}(A)=$ $\frac{(c-d)^{2}}{4}=1$ and $\operatorname{Var}(\tilde{R})=\frac{(c-d)^{2}}{12}=\frac{1}{3}$. The coefficients of Eq. (8.15) will be:

$$
\begin{aligned}
& -\frac{R^{2}}{2} \operatorname{Var}(\tilde{R})=-\frac{25}{6} \\
& \frac{3 R}{2} \operatorname{Var}(\tilde{R})=\frac{5}{2} \\
& -\left[1+R^{2}+\operatorname{Var}(\tilde{R})+\frac{R^{2}}{2} \operatorname{Var}_{f}(A)\right]=-\frac{233}{6} \\
& -1+R+\frac{R}{2} \operatorname{Var}_{f}(A)=\frac{13}{2}
\end{aligned}
$$

With these values, Eq. (8.15) becomes

$$
-\frac{25}{6} z^{3}+\frac{5}{2} z^{2}-\frac{233}{6} z+\frac{13}{2}=0
$$

By means of Cubic Equation Calculator, the above equation has the following solutions:

$$
\begin{aligned}
z_{1}= & 0.16869898557375712, z_{2}=0.21565050721312146 \\
& +i * 3.0332713058887566 \text { and } z_{3} \\
= & 0.21565050721312146 \\
& -i * 3.0332713058887566
\end{aligned}
$$

In conclusion, the optimal saving $s_{5}^{*}$ can be approximated by the unique real solution: $z_{1}=0.16869898557375712$.

\section{From data to optimal saving}

Consider a mixed model of optimal saving $(A, \tilde{R})$, in which the fuzzy number $A$ represents the labor income risk, and the random variable $\tilde{R}$ is the return of saving. Recall that $\tilde{R}=\tilde{r}+1$, where $\tilde{r}$ is the random variable that models the interest rate for saving. For this mixed model, we have the initial data:

- the sure income from period 0 is $y_{0}=100$;

- the labor income risk $A$ and interest rate for saving $\tilde{r}$ are known by the data in the table below:

\begin{tabular}{llllllllllll}
\hline$y_{1}$ & 12 & 13 & 16 & 17 & 10 & 13 & 18 & 15 & 12 & 14 & 12 \\
\hline$\tilde{r}$ & 0.1 & 0.3 & 0.5 & 0.2 & 0.2 & 0.4 & 0.6 & 0.1 & 0.4 & 0.2 & 0.2 \\
$\tilde{R}$ & 1.1 & 1.3 & 1.5 & 1.2 & 1.2 & 1.4 & 1.6 & 1.1 & 1.4 & 1.2 & 1.2 \\
\hline
\end{tabular}


- the consumer utility functions of the model: $u(x)=$ $v(x)=\ln (x)$;

- The weighting function has the form $f(\gamma)=2 \gamma$, for any $\gamma \in[0,1]$.

We intend that based on these data to determine the value of the indicator $K_{1}$, by using the approximation formula (6.16):

$$
K_{1}=2\left[\frac{u^{\prime}\left(y_{0}\right)-R u^{\prime}\left(y_{1}\right)}{u^{\prime \prime}\left(y_{0}\right)+R^{2} u^{\prime \prime}\left(y_{1}\right)}\right]^{2} \frac{\operatorname{Var}(\tilde{R})}{\operatorname{Var}_{f}(A)+\left[\frac{u^{\prime}\left(y_{0}\right)-R u^{\prime}\left(y_{1}\right)}{u^{\prime \prime}\left(y_{0}\right)+R^{2} u^{\prime \prime}\left(y_{1}\right)}\right]^{2} \operatorname{Var}(\tilde{R})} .
$$

In order to obtain the indicator $K_{1}$, we need to compute the values of all entities that appear in the right member of the above formula.

$R$ will be the mean of values of $\tilde{R}$ from the third line of the previous table and $\operatorname{Var}(\tilde{R})$ will be the statistical variance of this sequence of data. By using Excel, we obtain $R=1.29$ and $\operatorname{Var}(\tilde{R})=0.026$.

For obtaining $\operatorname{Var}_{f}(A)$, we will apply the sample percentile method of Vercher et al. (2007), so that from the string of values on the first line of the table to determine the trapezoidal fuzzy number $A$.

By the method of Vercher et al. (2007), we associate with a data set $z_{1}, \ldots, z_{n}$ a trapezoidal fuzzy number $A=(a, b, \alpha, \beta)$. Firstly we compute the percentiles $P_{5}, P_{40}, P_{60}, P_{95}$ associated with $z_{1}, \ldots, z_{n}$, and then, we consider the trapezoidal fuzzy number $A=(a, b, \alpha, \beta)$, defined by $a=P_{40}, a=P_{60}, \alpha=P_{40}-P_{5}$ and $\beta=P_{95}-P_{60}$.

Starting from the values on the first line of the table and applying the above formulas, using a calculation in Excel we find $P_{5}=11, P_{40}=13, P_{60}=14$ and $P_{95}=17.5$. Thus, the fuzzy trapezoidal number associated with this data set is $A=(a, b, \alpha, \beta)=(13,14,2,3.5)$. This fuzzy number will represent the labor income risk.

We know from Example 3.3.9 of Georgescu (2012) that the possibilistic expected value of the trapezoidal fuzzy number $A=(a, b, \alpha, \beta)$ has the form

$E_{f}(A)=\frac{a+b}{2}+\frac{\beta-\alpha}{2}$.

Thus applying this formula for $A=(13,14,2,3.5)$, we get $E_{f}(A)=13.75$. According to the construction of the mixed model $(A, \tilde{R})$, we have $y_{1}=E_{f}(A)$ (see Sect. 5), so in our case we have $y_{1}=13.75$.

Recall from Example 3.4.9 of Georgescu (2012) the form of the possibilistic variance of the trapezoidal fuzzy number $A=(a, b, \alpha, \beta)$ :

$\operatorname{Var}_{f}(A)=\frac{(b-a)^{2}}{4}+\frac{(b-a)(\alpha+\beta)}{6}+\frac{\alpha^{2}+\beta^{2}+\alpha \beta}{18}$
By applying this formula to the trapezoidal fuzzy number $A=(13,14,2,3.5)$, we obtain $\operatorname{Var}_{f}(A)=2.45$.

We remark that $u(x)=\ln (x), u^{\prime}(x)=\frac{1}{x}$ and $u^{\prime \prime}(x)=$ $-\frac{1}{x^{2}}$, and therefore, we get

$$
\begin{aligned}
u^{\prime}\left(y_{0}\right) & =u^{\prime}(100)=\frac{1}{100}=0.01, u^{\prime \prime}\left(y_{0}\right)=u^{\prime \prime}(100) \\
& =-\frac{1}{10^{2}}=-0.0001 \\
u^{\prime}\left(y_{1}\right) & =u^{\prime}(13.75)=\frac{1}{13.75}=0.072 ; u^{\prime \prime}\left(y_{1}\right)=u^{\prime \prime}(13.75) \\
& =-\frac{1}{13.75^{2}}=-0.0052
\end{aligned}
$$

We obtained the numerical values of all entities that appear in the expression in the right member of the approximation formula (6.16), so, by a straightforward computation, we get $K_{1}=9.41$.

Now we want to see whether for our mixed model the optimal saving level increases or decreases. In order to obtain this result, we need to verify the criterion offered by Theorem 6.1: $s_{5}^{*}-s^{*}>0$ iff $K_{1}<P R P\left(y_{1}, s^{*} R\right)$.

Remember from (6.1) that $P R P(y, x)=P R P\left(y_{1}, s^{*} R\right)$ $=s^{*} R \frac{u^{\prime \prime \prime}\left(y_{1}+s^{*} R\right)}{u^{\prime \prime}\left(y_{1}+s^{*} R\right)}$.

An approximate value of $s^{*}$ is obtained by using formula (6.15):

$s^{*}=\frac{u^{\prime}\left(y_{0}\right)-R u^{\prime}\left(y_{1}\right)}{u^{\prime \prime}\left(y_{0}\right)-R^{2} u^{\prime \prime}\left(y_{1}\right)}=\frac{u^{\prime}(100)-R u^{\prime}(13.75)}{u^{\prime \prime}(100)-R^{2} u^{\prime \prime}(13.75)}$

Applying this formula for $y_{0}=100, y_{1}=E_{f}(A)=$ 13.75 and $R=1.29$ we get $s^{*}=9.4158$, therefore $P R P\left(y_{1}, s^{*} R\right)=629.747$.

We see that $K_{1}<P R P\left(y_{1}, s^{*} R\right)$ so the presence of possibilistic risk represented by the trapezoidal fuzzy number $A=(13,14,2,3.5)$ generates the extra-saving.

In an analogous way from the start data, we can determine $K_{2}$ and then we can check the extra-saving criterion in Theorem 6.2 .

\section{Concluding remarks}

In the two saving models from the paper, the risk acts on the optimal saving level as a bidimensional mixed vector: if a component (labor income risk) is a random variable, then the other component (interest-rate risk) is a fuzzy number. To study the changes of the optimal saving produced by these risk combinations, several notions of precautionary saving are defined. The main results from the paper consist in proving some necessary and sufficient conditions on these precautionary savings when: 
(a) moving from the certain model to each of the two mixed models;

(b) moving from a single risk model to a mixed model.

The mentioned conditions are expressed according to the partial relative prudence index (Baiardi et al. 2014) and two new indicators $K_{1}, K_{2}$ (analogous to the indicator $K$ from Baiardi et al. 2014). In the expressions of $K_{1}, K_{2}$, we find the variances of the components of the mixed model, reflecting the way the combinations of the two risks (a probabilistic one, and a possibilistic one) affect the optimal saving level.

$K_{1}$ and $K_{2}$ are analogous to $K$, an indicator used in Magnani (2017) and Magnani and Menegatti (2015) as a threshold level for partial relative prudence index in the framework of probabilistic saving models with labor income risk and interest rate risk. In the mentioned works, it is studied the effect that the changes in the covariance between labor income risk and interest rate risk have on the values of $K$. A similar problem cannot be formulated in the case of mixed models in this paper because no connection is assumed between the components of a mixed vector of the form $(A, \tilde{R})$ or $(\tilde{y}, B)$. We do not know a notion of "covariance" between a random variable and a fuzzy number that reflects a relationship of interdependence between the uncertainty phenomena they shape. Such a notion could lead to new classes of mixed saving models and to the study of a problem of the type above.

In Gunning (2010), Vergara (2007), the effect on the optimal saving level of the four types of risk (labor income risk, wealth risk, asset risk and capital risk), all modeled by random variables, is analyzed. One asks the problem of studying saving models in which all these four types of risk are represented by fuzzy numbers, as well as models in which we have mixed risks obtained from the four risks mentioned above.

In Kaluszka and Krzeszowiec (2017), the Jensen-type operators were introduced, a notion that generalizes the expected utility operators from Chapter 5 of Georgescu (2012). With each Jensen-type operator, one associates an abstract EU-theory. In such a general framework, in Kaluszka and Krzeszowiec (2017) was obtained an Arrow-Pratt-type theorem, which generalizes both the Arrow-Pratt theorem from the classic theory of risk aversion (see, e.g., Eeckhoudt et al. 2005; Gollier 2004), and the possibilistic version of this result (see Georgescu 2012, 2009, 2011, 2014; Georgescu and Kinnunen 2016).

The paper Kaluszka and Krzeszowiec (2017) contains a great variety of Jensen-type operators. One of them offers another way to define a notion of mixed expected utility (see Ex. 10 of Kaluszka and Krzeszowiec (2017)). Then an interesting problem would be the study of some optimal saving models in the framework of EU-theory associated with a Jensen-type operator, or at least of optimal saving models corresponding to the operator of Example 10 of Kaluszka and Krzeszowiec (2017).
A series of works published lately have dealt with saving models in which there are multiple distributions of future incomes (cf. Osaki and Schlesinger 2013). These models have studied how an ambiguous attitude of the consumer influences the saving decisions. A question that arises is whether a relationship can be established between the ambiguity in these probabilistic saving models and the uncertainty of the fuzzy type. Can we define saving models in which an ambiguous attitude of the consumer is expressed through a multiple possibilistic distribution?

Acknowledgements The author would like to thank the reviewers for the recommendations and comments which led to an improved version of the paper.

Funding This study was not funded.

\section{Declaration}

Conflict of Interest Author Irina Georgescu declares that she has no conflict of interest.

Ethical approval All procedures performed in studies involving human participants were in accordance with the ethical standards of the institutional and/or national research committee and with the 1964 Helsinki Declaration and its later amendments or comparable ethical standards.

Ethical approval This article does not contain any studies with human participants or animals performed by any of the authors.

Informed consent Informed consent was obtained from all individual participants included in the study.

\section{References}

Baiardi D, Magnani M, Menegatti M (2014) Precautionary saving under many risks. J Econ 113(3):211-228

Baiardi D, Magnani M, Menegatti M (2019) The theory of precautionary saving: an overview of recent developments. Rev Econ Household. https://doi.org/10.1007/s11150-019-09460-3

Carlsson C, Fullér R (2001) On possibilistic mean value and variance of fuzzy numbers. Fuzzy Sets Syst 122:315-326

Carlsson C, Fullér R (2011) Possibility for decision. Springer, Berlin

Courbage C, Rey B (2007) Precautionary saving in the presence of other risks. Econ Theor 32:417-424

Drèze J, Modigliani F (1972) Consumption decision under uncertainty. J Econ Theory 5:308-355

Dubois D, Prade H (1988) Possibility theory. Plenum Press, New York

Dubois D, Foulloy L, Mauris G, Prade H (2004) Probability-possibility transformations, triangular fuzzy sets and probabilistic inequalities. Reliable Comput 10:273-297

Eeckhoudt L, Schlesinger H (2008) Changes in risk and the demand for saving. J Monetary Econ Elsevier 55(7):1329-1336

Eeckhoudt L, Gollier C, Schlesinger H (2005) Economic and financial decision under risk. Princeton University Press, Princeton

Eeckhoudt L, Rey B, Schlesinger H (2007) A good sign for multivariate risk taking. Manage Sci 53:117-124

Georgescu I (2009) Possibilistic risk aversion. Fuzzy Sets Syst 60:26082619 
Georgescu I (2011) A possibilistic approach to risk aversion. Soft Comput 15:795-801

Georgescu I (2012) Possibility Theory and the Risk. Springer, Berlin

Georgescu I (2014) Risk aversion, prudence and mixed optimal saving models. Kybernetika 50(5):706-724

Georgescu I, Kinnunen J (2016) Mixed models for risk aversion, optimal saving, and prudence. Fuzzy Econ Rev Int Assoc Fuzzy Set Manage Econ 21(2):47-70

Georgescu I, Kinnunen J (2020) The interest rate for saving as a possibilistic risk. Physica A 547(1):124460

Gollier C (2004) The economics of risk and time. MIT

Gunning JW (2010) Risk and saving: a taxonomy. Econ Lett 107(1):3941

Jappelli T, Pistaferri L (2017) The economics of consumption: theory and evidence. Oxford University Press, Oxford

Kaluszka M, Krzeszowiec M (2017) On risk aversion under fuzzy random data. Fuzzy Sets Syst 328(1):35-53

Kimball M. S. (1992) Precautionary motives for holding assets, new palgrave dictionary of money and finance, vol 3. MacMillan Press, London and Stockton Publishers, New York, pp 158-161

Kimball MS (1990) Precautionary saving in the small and in the large. Econometrica 58:53-73

Kimball MS, Weil P (2009) Precautionary saving and consumption smoothing across time and possibilities. J Money Credit Bank 41(2-3):245-284

Leland H (1968) Saving and uncertainty: the precautionary demand for saving. Quart J Econ 82:465-473

Li J (2012) Precautionary saving in the presence of labor income and interest rate risks. J Econ 106:251-266
Lucia-Casademunt AM, Georgescu I (2013) Connecting possibilistic prudence and optimal saving. Int $\mathbf{J}$ Interact Multim Artif Intell 2(4):38-45

Magnani M (2017) A new interpretation of the condition for precautionary saving in the presence of an interest-rate risk. J Econ 120:79-87

Magnani M, Menegatti M (2015) Precautionary saving and changes in risk correlation. Econ Bull 35(1):561-570

Nocetti CD (2016) Robust comparative statics of risk changes. Manage Sci 62:1381-1392

Osaki Y, Schlesinger H (2013) Precautionary saving and ambiguity. University of Alabama, July 25

Rothschild M, Stiglitz J (1971) Increasing risk II: economic consequences. J Econ Theory 3:66-84

Sandmo A (1970) The effect of uncertainty on saving decisions. Rev Econ Stud 37:353-360

Vercher E, Bermudez JD, Segura JV (2007) Fuzzy portfolio optimization under downside risk measures. Fuzzy Sets Syst 158:769-782

Vergara M (2007) Precautionary saving: a taxonomy of prudence. Econ Lett 15:18-20

Vergara M, Bonilla CA (2021) Precautionary saving in mean-variance models and different sources of risk. Econ Model 98:280-289

Zadeh LA (1978) Fuzzy sets as a basis for a theory of possibility. Fuzzy Sets Syst 1:3-28

Publisher's Note Springer Nature remains neutral with regard to jurisdictional claims in published maps and institutional affiliations. 